\title{
CLINICAL OUTCOMES OF DIFFERENT TEMPOS OF MUSIC DURING EXERCISE IN CARDIAC REHABILITATION PATIENTS
}

\author{
A Thesis \\ Submitted to the Graduate Faculty \\ of the \\ North Dakota State University \\ of Agriculture and Applied Science
}

\author{
By \\ Jarad Steven Miller \\ In Partial Fulfillment of the Requirements \\ for the Degree of \\ MASTER OF SCIENCE \\ Major Department: \\ Health, Nutrition and Exercise Sciences
}

November, 2014

Fargo, North Dakota 


\section{North Dakota State University \\ Graduate School}

Title

Clinical Outcomes of Different Tempos of Music during Exercise in

Cardiac Rehabilitation Patients

By

Jarad Steven Miller

The Supervisory Committee certifies that this disquisition complies with North Dakota State

University's regulations and meets the accepted standards for the degree of

MASTER OF SCIENCE

SUPERVISORY COMMITTEE:

Donna J. Terbizan

Chair

Bryan Christensen

Mary Wright

Approved by Department Chair:

$11 / 12 / 14$

Margaret Fitzgerald

Date

Department Chair 


\begin{abstract}
This study examined the effects of stimulating and sedative music on ratings of perceived exertion (RPE), heart rate (HR), blood pressure (BP), and feeling status during exercise in cardiac rehabilitation (CR) patients. Twenty-two male and female older adults age $64 \pm 8.0$ y currently enrolled in phase III CR completed the study. Repeated measures crossover designs guided data collection. The manipulated independent variable was music condition (sedative, stimulating, and non-music control). The dependent variables were RPE, BP, HR, and feeling status with each represented by four repeated measures ANOVAs over time via SAS 9.3. Data analysis indicated significant differences for all exercise related variables besides BP. While standardizing the exercise, we observed that sedative music is the best choice to manipulate for decreases in RPE ( $p=.0019)$, increases in feeling status $(p=.0192)$, and decreases in HR (p<.0001). Stimulating music would only be the correct choice to observe increases in HR $(\mathrm{p}<.001)$
\end{abstract}




\section{ACKNOWLEDGEMENTS}

I would like to express my appreciation to Dr. Donna Terbizan for her guidance and support throughout this project, and extend my gratitude to my committee members Dr. Bryan Christensen and Dr. Mary Wright for their time, feedback, and direction provided. I would also like to acknowledge Sanford Health for allowing me to utilize their patients and facilities. I owe the deepest thanks and appreciation to close family, friends, and mentors over the years that have continuously supported and enabled me to be where I am today. I am so grateful to have these people in my life and hold them all very close in my heart, as they are the reason and purpose for all my success and achievements. 


\section{TABLE OF CONTENTS}

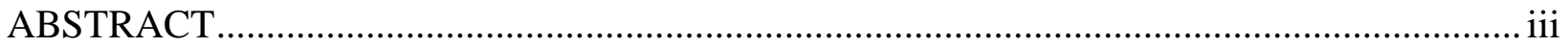

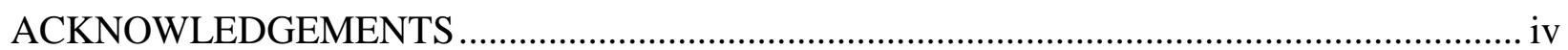

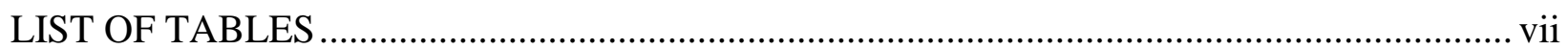

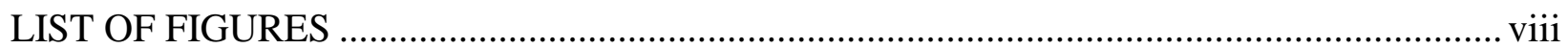

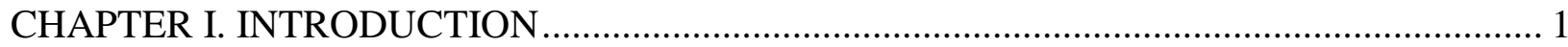

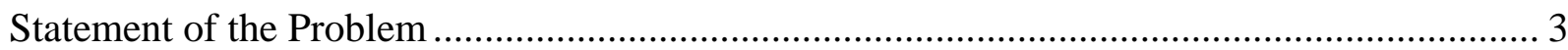

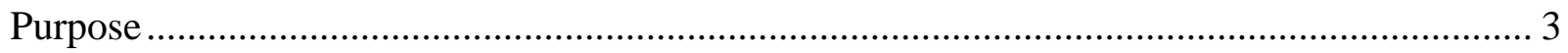

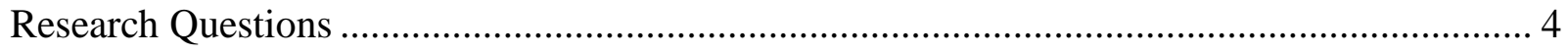

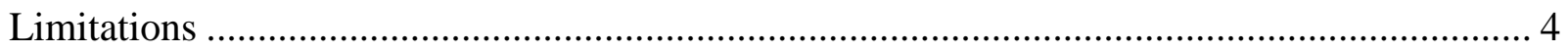

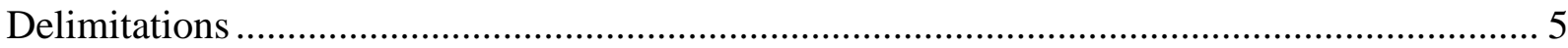

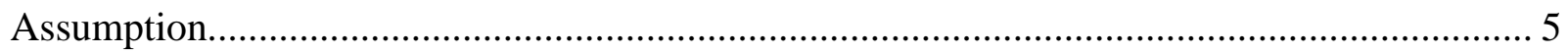

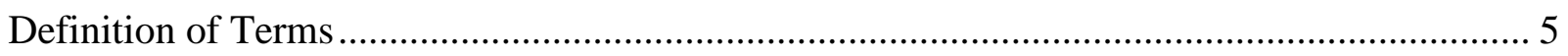

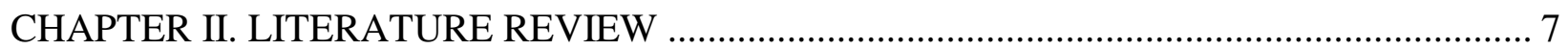

Predicting the Effects of Music when Combined with Exercise (Psychophysical Effects)........ 7

Effects of Music when Combined with Exercise _.............................................................. 10

Common Uses and Measurements of Music's Effects without Exercise................................. 18

Use of Music in Inpatient and Outpatient Cardiac Care Settings ............................................ 21

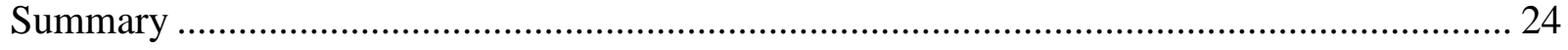

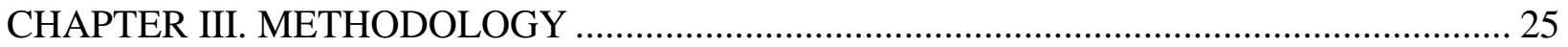




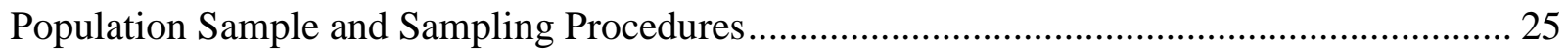

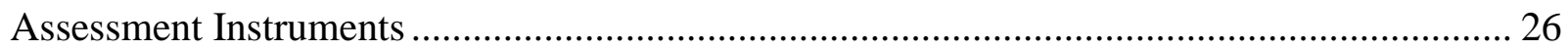

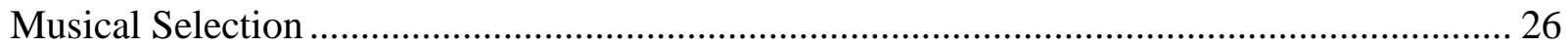

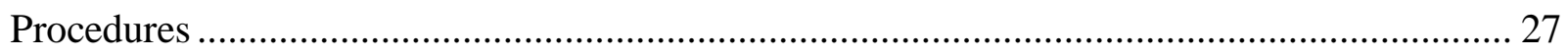

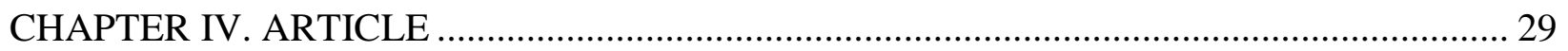

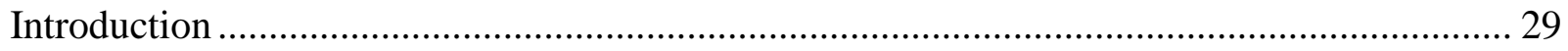

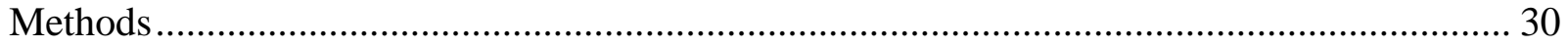

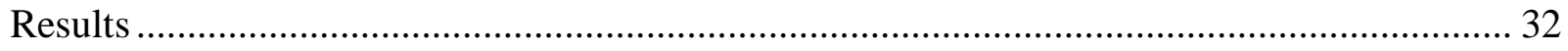

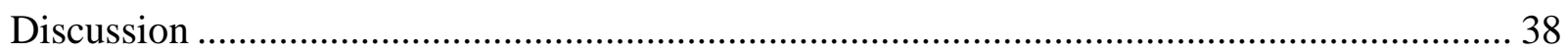

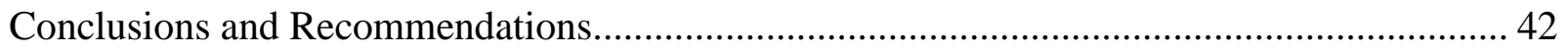

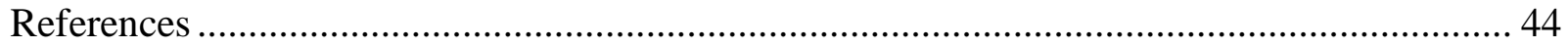

CHAPTER V. SUMMARY, CONCLUSIONS, AND RECOMMENDATIONS FOR

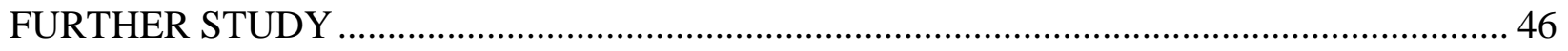

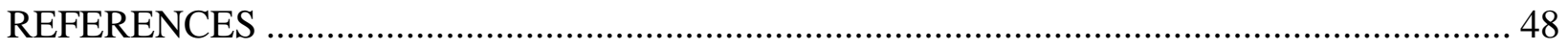

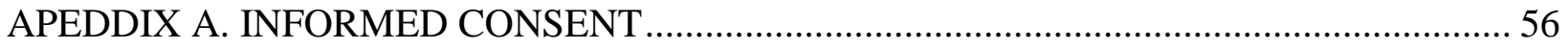

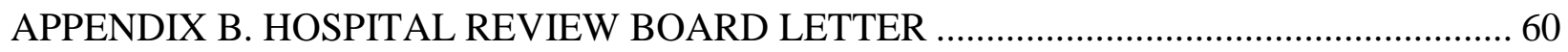

APPENDIX C. INSTITUTIONAL REVIEW BOARD LETTER ....................................... 61

APPENDIX D. RATING OF PERCEIVED EXERTION SCALE …...................................... 64

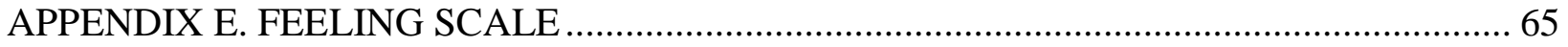




\section{LIST OF TABLES}

Table $\quad \underline{\text { Page }}$

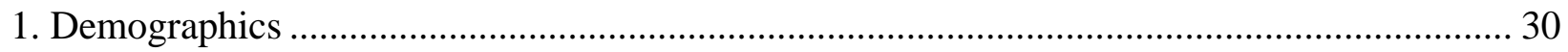

2. Means with Standard Deviations .................................................................................. 33 


\section{LIST OF FIGURES}

Figure $\quad \underline{\text { Page }}$

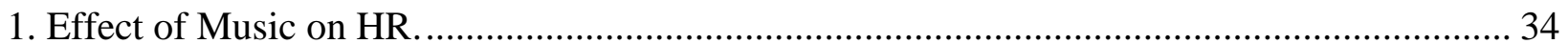

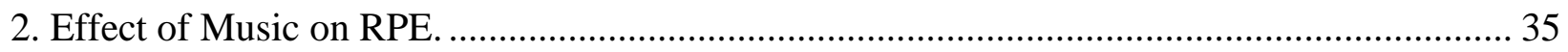

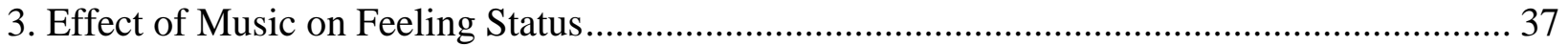




\section{CHAPTER I. INTRODUCTION}

According to the National Vital Statistics Reports (2012), cardiovascular disease (CVD) is the number one killer in America. In 2011, CVD accounted for approximately $24 \%$ of all deaths in the United States (Hoyert \& Xu, 2012). Cardiac rehabilitation (CR) programs are aimed at combating this disease with the intent of slowing its progression or even reversing the symptoms. Phase III of any CR program is referred to as the maintenance phase. This phase focuses on improving physiological and psychological parameters in order for patients to maintain long-term healthy lifestyle changes and learn to self-manage their cardiovascular health. The central component of this phase which contributes to improvement in physiological parameters (such as increased physical endurance and enhance cardiopulmonary function) is exercise (Emery, Hsiao, Hill, \& Frid, 2003). Even moderate-intensity exercise if done regularly and long term can help reduce risk factors and symptoms of CVD (Fletcher et al., 1996).

CR specialists may further be able to expand upon the benefits of phase III CR programs by incorporating music into their patient's exercise programs. Researchers have determined that listening to music during exercise has presented beneficial effects in the physical activity, sport and exercise environment for years (Karageorghis \& Priest, 2012a, 2012b; Karageorghis \& Terry, 1997). Many aspects of music have been studied during exercise; including the effect of music tempo (Iwanaga, 1995; Karageorghis, Jones, \& Low, 2006; Pujol \& Langenfeld, 1999; Waterhouse, Hudson, \& Edwards, 2010), synchronous and asynchronous music (Anshel \& Marisi, 1978; Crust, 2008; Karageorghis et al., 2011), and preferred or non-preferred music (Nakamura, Papini, Pereira, Nakamura, \& Kokubun, 2010) on numerous dependent variables such as: cycling performance, treadmill performance, swimming performance, and physiological and psychological measures. A large portion of the research focused on music used during 
exercise within high school to college age populations for the purposes of examining performance variables. However, studies observing the effects of music during exercise within CR populations are limited (Emery et al., 2003; Murrock, 2002; MacNay, 1995). This population warranted further examination of more clinical outcomes of listening to music during exercise (blood pressure [BP], heart rate [HR], rating of perceived exertion [RPE], and feeling status) as music may continue to positively influence the medical field as well.

For example, feeling status can essentially be viewed as how one feels in relation to music that's being played (Hardy \& Rejeski, 1989). One way to observe whether individuals prefer the type of music they are exposed to is to determine their feeling status while listening to music. A measure that researchers can use for measuring feeling status is Rejeski's (1985) 11point feeling scale. Higher scores on the feeling scale means an increase in the amount of pleasure associated with the music. Compliance with an exercise program depends on the degree of pleasure associated with exercise. The more pleasure perceived, the more likely the exercise will be repeated to promote exercise compliance (Murrock, 2002). Therefore, improvement in feeling status during exercise will also enhance compliance to the exercise program (Murrock, 2002).

Currently, music is not used in our local CR program despite the potential benefits found within a mix of older populations as well as cardiac patients, such as decreased RPE (MacNay, 1995), increased HR (Metzger, 2004), increased feeling status (Murrock, 2002), decreased systolic BP (Byers \& Smyth, 1997), and improvements in overall quality of life (Lee, Moon Fai, \& Mok, 2010). Adding to the mix however, other researchers suggest that music does not alter BP (Sendelbach, Halm, Doran, Miller, \& Gaillard, 2006; Özer, Karaman Özlü, Arslan, \& Günes, 
2013), HR (Nilsson, 2009; White, 1999; Özer et al., 2013), or RPE (Murrock, 2002) in cardiac patients.

\section{Statement of the Problem}

According to the literature, two main reasons arose for the use of music in an outpatient CR program. The first has been to reduce RPE during exercise, and the second is to increase the feeling status of patients in order to increase compliance (Murrock, 2002; MacNay, 1995). The effects of music on physiological and psychological variables of RPE, HR, BP, and feeling status during exercise in cardiac patients are controversial thus far within the literature. A factor that can alter the effect of music on RPE and feeling status during exercise is the tempo of music. No evidence exists for the effect of music tempo on those variables during exercise in CR patients. Researchers have recommended that a study utilizing different music tempo in CR patients may help determine if the tempo is the cause for the controversy in the literature on RPE and feeling status (Murrock, 2002). In the present study, sedative and stimulating music (effect of music tempo) was used to further examine the clinical outcomes to present a clearer consensus among the research in CR populations. It is important to note that along with music tempo; HR, BP and arrhythmias noted during exercise can also affect RPE and feeling status. No research was found to have documented these variables in addition to RPE and feeling status.

\section{$\underline{\text { Purpose }}$}

The purpose of this study was to examine the effects of stimulating and sedative music on RPE, HR, BP, and feeling status during exercise in Phase III CR patients. One objective was to see if there were differences in RPE or feeling status during exercise based on the tempo of music. If either type of music used in this study decreased RPE, or increased feeling status in CR patients, this would warrant the use of music in CR programs for exercise compliance. 
Furthermore, if a patient's RPE was lowered while exercising to music, exercise would be done more effortlessly, which could lead to physiological improvements (Murrock, 2002). Another objective was to record any differences in HR and BP between conditions. Knowing how different tempos of music affect $\mathrm{HR}$ and $\mathrm{BP}$ in $\mathrm{CR}$ patients may then warrant the use for music for a specified outcome for the CR specialists. CR specialists often find it difficult to adjust the exercise routine for the patients because of the "comfort of the routine" for patients; whether it be adjusting speed, incline, MET level, exercise equipment, etc. This study presented other possible pathways to increase or decrease HR and/or BP during exercise without adjusting the exercise routine.

\section{$\underline{\text { Research Questions }}$}

1. Are there any significant differences in RPE between conditions during a standardized treadmill exercise in CR patients?

2. Are there any significant differences in feeling status between conditions during a standardized treadmill exercise in CR patients?

3. Are there any significant differences in HR between conditions during a standardized treadmill exercise in CR patients?

4. Are there any significant differences in BP between conditions during a standardized treadmill exercise in CR patients?

\section{$\underline{\text { Limitations }}$}

1. Subjects may be on medications that can affect BP and HR that only allow them to reach a certain limit.

2. The number of subjects available may vary due to only being able to recruit from Sanford Health in Fargo, ND. 
3. The musical selection chosen will be preferred by the group as a whole and not entirely individually selected.

\section{$\underline{\text { Delimitations }}$}

1. Subjects must be enrolled in phase III cardiac rehabilitation program at Sanford Health and be between the ages of (55-80).

2. We will implement subject blinding to protect from subject bias.

\section{Assumption}

1. That participants will accurately report RPE and feeling status.

\section{Definition of Terms}

Rating of Perceived Exertion - An indicator of the degree of physical strain (Borg, 1982). The scale consists of a range of numbers (6-20) that increase linearly to the subject's perceived effort (e.g., 7/very very light, 9/very light, 11/fairly light, 13/somewhat hard, 15/hard, 17/very hard, and 19/very very hard).

Sedative Music- Slower paced, more relaxing music approximately 90-100 bpm (C. I. Karageorghis et al., 2011).

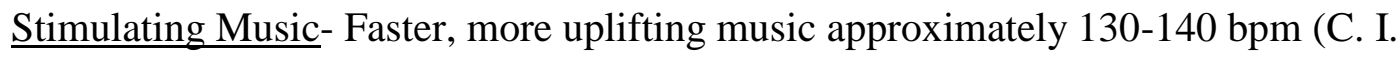
Karageorghis et al., 2011).

Synchronous Music- Music that is synched with any physical movement (C. I. Karageorghis \& Priest, 2012a).

Asynchronous Music- Background music, music that is not coordinated with movement (C. I. Karageorghis \& Priest, 2012a).

Blood Pressure- Blood Pressure is the pressure of the blood within the arteries recorded by two numbers. Systolic BP is he higher of the two numbers which measures the pressure in the 
arteries when the heart beats (when the heart muscle contracts). Diastolic BP is the lower of the two numbers which measures the pressure in the arteries between heartbeats (when the heart muscle is resting between beats and refilling with blood) (National Heart, Lung, and Blood Institute, 2012).

Heart Rate- The number of heartbeats per unit of time, expressed as beats per minute (National Heart, Lung, and Blood Institute 2012). 


\section{CHAPTER II. LITERATURE REVIEW}

The purpose of this study was to examine the effects of stimulating and sedative music on RPE, HR, BP, and feeling status during exercise in CR patients.

Predicting the Effects of Music when Combined with Exercise (Psychophysical Effects)

\section{Motivational Qualities of Music}

There can be many benefits of listening to music during exercise. Karageorghis and Priest (2012a) suggest that the benefits of listening to music during exercise performance are largely attributed to the motivational qualities of the music, qualities such as reduced RPE, improved mood, enhanced performance, and enhanced exercise adherence. Motivational qualities of music are best defined as the beneficial outcomes of listening to music, including the control of arousal, reduced perceptions of exertion, and improved mood (Karageorghis \& Priest, 2012a). These authors presented four main factors that contribute to motivational qualities of music: (1) rhythm response (beats per minute) (2) musicality (tune) (3) cultural impact (4) and association. These 4 factors are thought to promote long-term benefits such as increased exercise adherence among participants and a more effective pre-event routine for elite athletes. It was suggested that if a person experiences any of these factors or motivational qualities from the type of music they listen to, it may lead to enhanced performance or a more enjoyable bout of exercise (Karageorghis \& Priest, 2012a).

Karageorghis and Priest (2012a) also suggest that scientists should rate the motivational qualities of music. The higher the motivational quality of the music, the more positive moods and feeling states are realized. To rate the motivational qualities of music, researchers have used the Brunel Music Rating Inventory (BMRI) developed by Karageorghis, Terry, and Lane in 1999. The four main motivational qualities of music previously discussed were initially validated through the use of this instrument. However, this tool was improved upon to enhance its 
psychometric (interpretation of quantitative tests for the measurement of psychological variables) properties and usability in 2006 (BMRI-2) (Karageorghis, Priest, Terry, Chatzisarantis, \& Lane, 2006). The outcome of this scale is to allow researchers to better predict of psychophysical responses (RPE) to music during submaximal exercise (Karageorghis et al., 1999). Rating of Perceived Exertion (RPE)

One psychophysical effect of music is the subjective perception of physical effort or fatigue. A psychophysical measure of subjective perception of physical effort or fatigue is Borg's RPE scale (Borg, 1982). Here, RPE has been defined as an the indicator of the degree of physical strain (Borg, 1982). The version of this scale utilized in CR programs consists of a range of numbers (6-20) that increase linearly to the subject's perceived effort (e.g., 7/very very light, 9/very light, 11/fairly light, 13/somewhat hard, 15/hard, 17/very hard, and 19/very very hard). The overall perceived exertion rating integrates various information, including signals from the working muscles and joints, from cardiovascular and respiratory functions, and from the central nervous system (Borg, 1982). All of this information is then combined into a "Gestalt" or configuration of perceived exertion (Borg, 1982). Certain authors have found that music plays a key role in lowering RPE during exercise (Boutcher \& Trenske, 1990; Mohammadzadeh, Tartibiyan, \& Ahmadi, 2008a; Potteiger, Schroeder, \& Goff, 2000; Szmedra \& Bacharach, 1998; Yamashita, Iwai, Akimoto, Sugawara, \& Kono, 2006), two of which used cycle ergometer tests to analyze the effect of music on RPE. Both authors that used cycle tests found that RPE was lower while listening to music versus a no-music control. The same result held true in participants during treadmill exercise (Mohammadzadeh et al., 2008; Szmedra \& Bacharach, 1998). Although each of these authors suggested music does lower RPE, it only significantly lowers RPE at low to moderate intensities (Boutcher \& Trenske, 1990; Mohammadzadeh et al., 
2008; Yamashita et al., 2006). An explanation for this effect could be that participants may only react to the effects of music as a passive distractor during treadmill exercise (Bauldoff, Hoffman, Zullo, \& Sciurba, 2002; Bharani, Sahu, \& Mathew, 2004). Once the exerciser reaches high workloads or high intensities the individual may not pay attention to the music anymore and focus their attention back towards the task, consequently eliminating effects of the music. With this information, researchers can expect that when music is used at low intensities as a distractor, it may lower RPE, but once the physiological effects of the exercise reaches a higher intensity (which is variable to each individual) the distractor may not be beneficial anymore.

\section{Ergogenic Effects of Music}

When an individual focuses on music during low to moderate intensities of exercise, it can result in an ergogenic effect. An ergogenic effect during exercise is evident when music improves exercise performance by either delaying fatigue or increasing work capacity (Karageorghis \& Priest, 2012a). Music has even been commonly referred to as a legal performance enhancer (Karageorghis \& Priest, 2012a). However, only minor ergogenic effects were found within a CR population (MacNay, 1995). MacNay (1995) asked 4 CR patients to exercise to a preferred music condition and a no-music control condition for 15 accumulative exercise sessions. Results indicated that subjects experienced a non-significant decrease in RPE (MacNay, 1995). No significant changes in RPE or performance were reported (MacNay, 1995). More recently, Murrock (2002) also compared exercise sessions with and without music in cardiac rehabilitation patients. Results were consistent with MacNay’s (1995) findings; no change in RPE or performance was reported. From these studies, we can conclude that music may not improve RPE or performance in CR patients, or that the methodological designs need to be further inspected as to why results are not consistent with other research. 


\section{Effects of Music when Combined with Exercise}

\section{Effects of Music on Heart Rate}

While listening to music during exercise, certain physiological (HR and BP) and psychological (emotion and affect) changes occur. One of the mechanisms that alters HR response is the tempo of music. Listening to stimulating music can result in the aroused physiological response of increased HR (Lingham \& Theorell, 2009). These researchers found that at rest, stimulating music increases heart rate and respiratory rate when participants get the chance to select their own piece of stimulating music, suggesting that this response could be due to a feeling of joy, elated mood and energy while listening to stimulating music. In the same study, increased feelings of joy and energy were also observed when individuals selected their own piece of sedative music (Lingham \& Theorell, 2009). However, instead of raising HR as shown with stimulating music, sedative music induced feelings of calmness and relaxation, which in this case lowered HR. Consistent with Lingham and Theorell's (2009) study, two other studies noted that sedative music has been shown to lower participant's HR during treadmill exercise (Ghaderi, Rahimi, \& Azarbayjani, 2009; Hepler \& Kapke, 1996). Both studies were consistent with the conclusion of Lingham and Theorell (2009) that sedative music reduces arousal which, in turn, reduces HR.

In contrast to these findings, Schwartz (1990) found that music did not influence HR. The researchers asked 10 untrained women and 10 untrained men to participate in a submaximal cycle ergometry test to determine RPE and HR between a stimulating music condition and a nomusic condition. They observed no differences in RPE or HR between conditions. The difference between finding a change in HR and not finding a change in HR may be due to methodological design. A limitation to this study is that the author did not allow participants to select their own 
piece of music. Participants may not have enjoyed the piece of music selected by the experimenter, which may have altered the effects of sedative music (reduced HR) that are consistent within other studies (Schwartz, 1990).

The conflict in HR response from listening to music may be due to the fact that there is a difference between how music alters arousal (which may increase or decrease HR) and how music tempo may alter HR (Iwanaga, 1995). Iwanaga (1995) suggests that individuals prefer music tempo similar to what their heart rate is at the time they choose their tempo preference during exercise. For example, if an individual's heart rate is $100 \mathrm{bpm}$ during exercise and the individual gets the chance to choose tempo of music, the tempo they will choose will be approximately $100 \mathrm{bpm}$. If subjects were to raise their HR during exercise, they would prefer higher tempo music while performing higher intensity exercise. In summary, differences in music tempo may cause certain arousals, which may increase or decrease HR; these changes may be different between every individual. Furthermore, individuals prefer music tempo close to their actual HR at rest and during exercise, which means when experimenters determine the musical selection, research shows that the most significant levels of arousal (changes in heart rate) will be reached when the level of intensity correlates with an individual's HR (Iwanaga, 1995).

\section{Effects of Music on Blood Pressure}

Music's effect on blood pressure (BP) was examined in hospital patients due to support from the literature. Hamel (2001) and Vaajoki, Kankkunen, Pietilä, and Vehviläinen-Julkunen, (2011) suggest that listening to music will lower systolic BP pre-surgery. Both studies used a quasiexperimental pre-test/post-test design without an exercise component. In each study, the music group experienced significantly lower systolic BP pre-surgery compared to the no-music control group (Hamel, 2001; Vaajoki et al., 2011). However, numerous studies do not support 
the theory that music may lower BP (Barnason, Zimmerman, \& Nieveen, 1995; Elliott, 1994; White, 1992; Sendelbach et al., 2006; Zimmerman, Pierson, \& Marker, 1988). Barnason et al. (1995), Elliot (1994), Sendlebach et al. (2006), and Zimmerman et al. (1988), each studied the effects of music therapy on BP; all authors found no significant differences in blood pressure pre- and post-surgery under any music condition. The controversy within the literature may be due to the fact that BP may consistently change over time, which can be a limitation to research within this area. It is well known that there are numerous factors which may affect individual's blood pressure over time. Common factors that affect BP over time include diet, exercise, and stress (Rosch, 1994). It may be difficult to control for these factors when studying the long term effects of listening to music on BP. During relatively shorter pretest/posttest studies examining the effect of music on resting BP, those variables may be easily controlled for, but it is still unknown as to why there are inconsistencies within the literature. Only one study examined the effects of music on BP during exercise in cardiac rehabilitation patients. Emery et al. (2003) asked cardiac rehabilitation subjects to complete two treadmill exercise sessions scheduled one week apart. During one of the exercise sessions participants listened to music, and during the other, participants listened to a blank tape. Emery et al. (2003) concluded that there were no significant differences in blood pressure between listening to music and not listening to music during exercise.

\section{Psychological Effects of Music}

In addition to music's physiological effects, individuals can also experience psychological effects from listening to music. A psychological effect of music is expressed by the way in which music can influence factors such as emotion, affect (feelings of pleasure or displeasure) and/or behavior (Karageorghis \& Priest, 2012a). These factors can affect how 
individuals perform their activity or respond to the kind of music chosen by the experimenters. For example, when looking at sedative music, the common theory would be that sedative music may lower HR. However, if an individual dislikes a certain type of sedative music it may cause an increase in HR from feelings of displeasure. One way to observe whether individuals prefer the type of music they are exposed to is to determine their feeling status while listening to music. A measure that researchers can use for measuring feeling status is Rejeski's (1985) 11-point feeling scale. The feeling scale ranges from +5 to -5 with verbal anchors of $+5=$ very good, $+3=$ good, $+1=$ fairly good, $0=$ neutral, $-1=$ fairly bad, $-3=$ bad, $-5=$ very bad. Validity of this scale was demonstrated by Hardy and Rejeski (1989). These researchers believe feeling status to be a more sensitive measure than RPE for how a person feels during exercise (Hardy \& Rejeski, 1989). For this reason, it should be used along with the RPE scale. Furthermore, one approach to enhance compliance to an exercise program would be to make exercise more enjoyable. If a type or piece of music can make exercise more enjoyable for CR patients, there may be additional implications for use of the feeling scale to determine what kind of music may enhance compliance.

Another psychological change that can influence performance is how subjects may react when they know music is the main variable of the experiment (Mohammadzadeh et al., 2008). If participants become aware that the purpose of the experiment is to assess the effects of music in relation to exercise, it may produce testing bias. This affect can either be in favor of the research hypothesis or in favor of the null hypothesis depending on the subject's reactions. Blinding subjects to the experiment may be an alternative route to avoid undesirable behavioral change. In addition, blinding experimenters to the type of music may also reduce any experimenter bias. 
Effects of Synchronous vs. Asynchronous Music

Different types of music cause different outcomes during exercise. Researchers

knowingly choose different pieces of music depending on what it is they want to observe. For example, researchers have utilized synchronous music in relation to exercise for observing ergogenic effects, such as delay in fatigue and improved endurance (Anshel \& Marisi, 1978; Simpson \& Karageorghis, 2006). The theory is that synchronizing music with movement will facilitate better performance. Anshel and Marisi (1978) suggest that music synchronized with physical movement causes positive effects on a subject's ability to endure workloads. In addition, physical endurance may be enhanced if movement is coordinated with musical stimuli (Anshel \& Marisi, 1978). In this study subjects showed elevated endurance-time scores (in minutes) while listening to synchronous music vs. not listening to music while on a bicycle ergometer (12.61 \pm 5.49 minutes vs. $9.92 \pm 4.27$ minutes, respectively). It should also be noted that the type of music used in this study was stimulating. Although results of this study did prove statistically significant differences ( $\mathrm{p}<0.05)$, it is unclear to whether physical endurance was enhanced by the synchronous music or the stimulating music. There is no distinction to which had greater effect over the other: the only conclusions taken from Anshel and Marisi's (1978) study are that stimulating-synchronous music may enhance endurance. Adding to the discovery that synchronous music may improve performance, many years later Simpson and Karageorghis (2006) tested the hypothesis that synchronous music would result in better performance in a 400$\mathrm{m}$ sprint. Participants ran faster with synchronous music than a no-music control, providing further evidence that when music is synched with movement it increases performance.

Asynchronous music also causes certain affects during exercise. Although asynchronous music has not been found to improve performance, it may play a role in lowering perceived 
intensity. Boutcher and Trenske (1990) observed the effects of asynchronous music on heart rate, perceived exertion, and affect. They found that the music condition produced lower RPE and a significantly greater level of affect (in this case, feelings of pleasure). However, no changes were seen in performance (Boutcher \& Trenske, 1990).

Years later, a different approach was taken to examine use of asynchronous music (Crust, 2008). The aim of the study was to identify the importance of asynchronous music during a circuit training exercise. After exercising to asynchronous music, participants rated the importance of 13 components of asynchronous music in relation to exercise enjoyment. The most important components identified by both male and female participants were tempo, beat, and rhythm in determining enjoyment of the class (Crust, 2008). In addition to finding the true importance of the use of asynchronous music, Crust's (2008) study also added to the literature on how the use of asynchronous music can alter enjoyment levels, which is an important psychological factor that may alter performance.

Effects of Sedative vs. Stimulating Music

Sedative and stimulating music also affects an exerciser in different ways. To clarify terms, a tempo of 95-100 beats per/min (bpm) has been considered sedative music (slow), and a tempo between 130-140 bpm has been considered stimulating music (fast) (Karageorghis et al., 2011). Stimulating music has been shown to improve cycling performance (Anshel \& Marisi, 1978; Nakamura et al., 2010), and maximal effort runs on a treadmill (Barwood, Weston, Thelwell, \& Page, 2009). Improvements in exercise performance may be due to the fact that stimulating music is a strong motivator for performance when utilized at moderate to high intensity exercise. The rationale is that the up tempo beat of stimulating music carries certain 
motivational factors, which contribute to improved performance during exercise (Karageorghis \& Priest, 2012a).

Sedative music, however, has not been associated with improvements in performance. Two authors observed how sedative music affects performance during treadmill running (Ghaderi et al., 2009; Hepler \& Kapke, 1996). To observe the effects of sedative music on performance, Helper and Kapke (1996) asked participants to run on a treadmill at a moderate intensity, while Ghaderi et al. (2009) asked participants to run on a treadmill at a high intensity. In both circumstances, sedative music did not improve performance. The results of their study could lead to the belief that if researchers want to best utilize sedative music in a non-exercise setting, then it would be the right choice to reduce anxiety and stress, and to improve mood as found in other research (White, 1992). If researchers want to improve performance during exercise, sedative music would only serve a prominent role in a slower paced class such as yoga (North \& Hargreaves, 1996), which focuses on relaxation of the body and mind among other things. In this type of setting, participants may prefer low to moderate tempo music opposed to stimulating music.

Effects of Music on Trained vs. Untrained Individuals

The effects of music also vary between trained and untrained individuals. Two studies observed the difference in response to music on performance between trained and untrained individuals, both of which suggest that music is more beneficial for the untrained individual (Brownley, McMurray, \& Hackney, 1995; Mohammadzadeh et al., 2008). The first study to examine this difference was conducted in 1995. Brownley et al. (1995) examined differences in feeling states (measured using the Rejeski (1989) feeling scale) by testing the effects of sedative and stimulating music in low-, moderate-, and high-intensity exercise between trained and 
untrained subjects. In the 16 volunteers who participated in the study, the authors found that untrained participants showed more positive feeling states during low intensity exercise in response to music than trained participants (Brownley et al., 1995).

Years later, Mohammadzadeh et al. (2008) tested the effects of music on endurance and RPE in both trained and untrained college students during a Bruce treadmill test. Both groups showed higher endurance under the music condition versus the no-music control. In addition, this study showed that untrained individuals exhibited lower RPE than trained individuals while listening to music during exercise, which provided further evidence to suggest that music is more beneficial for the untrained participant. A logical suggestion for this occurrence could be that trained individuals may have a better understanding of how their bodies function during exercise and have a good sense for how hard they are working whether music is present or not. Untrained individuals may not be as familiar with their bodies during exercise, a distractor such as music may be enough to take their mind off an exercise they may have otherwise found difficult. These studies indicate that in cases of low intensity exercise, untrained individuals experience greater benefits from listening to music during exercise.

Effects of Music When Introduced Before Exercise

The congruence of music with the task should also be considered for how it may affect performance. According to the literature, research typically focuses on the introduction of music for pre-task performance enhancement. Hall and Erickson (1995) examined the effect of preparatory arousal by listening to music pre-task (before performance). The authors used stimulating music (the Rocky theme) in comparison to a no-music control condition (waiting for 1 minute) prior to a 60 meter dash. The music condition resulted in faster times than the nomusic control condition, an effect of which may be due to the motivational qualities of 
stimulating music (Hall \& Erickson, 1995). Adding to Hall and Erickson's (1995) results, a combination of music and video has been combined to improve performance pre-task (Barwood et al., 2009). The authors hypothesized that a combination of a music and video intervention would include more external stimuli to induce a dissociative attentional strategy and increase exercise tolerance, testing their hypothesis by examining distance covered during a 15-minute treadmill maximal effort run. Their hypothesis was supported when subjects covered more distance after listening to a motivational music and video combination, suggesting that if music is introduced pre-task, it can improve performance (Barwood et al., 2009). Although results of this study are significant, it is unclear whether the findings were due to the music or the video. The authors only know how both variables affect performance when combined. However, regardless of what variable had greater affect, the fact remains that the use of music pre-task can improve performance.

\section{Common Uses and Measurements of Music's Effects without Exercise}

\section{Effects of Music on Well-Being and Quality of Life}

The use of music has also been studied without accompanying exercise. According to the literature, the most common health outcomes researched here include how music improves wellbeing and quality of life (Laukka, 2007; Lee et al., 2010; Murrock \& Higgins, 2009), and how music reduces stress and anxiety (Barnason et al., 1995; Chang, Peng, Wang, \& Lai, 2011; Labbé, Schmidt, Babin, \& Pharr, 2007; Sendelbach et al., 2006; Zimmerman et al., 1988). From a healthcare point of view, the goal of a healthcare program is not only to extend a patient's life but also to raise this patient's quality of life, which has become an important measure of health outcomes within hospital patients. According to the literature, there are six measures of wellness that can be examined to interpret quality of life of older adults from listening to music: self- 
awareness, positive relations with others, autonomy, environmental mastery, purpose of life, and personal growth (Laukka, 2007). The six measures of wellness were examined via survey data. According to these measures, listening to music was found to be a common leisure activity and a frequent source of positive emotions for older adults (Laukka, 2007). In addition, those who listen to music may also find that positive emotions are among the most frequent felt in response to music, and that the positive emotions may be associated with psychological well-being (Laukka, 2007). However, it should be noted that these results were based on a relatively small sample size for survey data. The combination of self-reported data along with a small sample size may limit the generalizability for entire populations.

To support the effects of music on health outcomes of older adults, authors also turned to the SF-36 physical and mental health summary scale to measure quality of life outcomes from listening to music (Lee et al., 2010). This measure comprises 36 items organized into eight scales: physical functioning, physical problem limitations, bodily pain, general health, vitality, social functioning, emotional problem limitations, and mental health (Ware \& Kosinski, 2001). Using the SF-36, statistically significant improvements in quality of life scores for older adults were seen when listening to music compared to not listening to music over a four-week time period (Lee et al., 2010). In addition, the authors of this study also suggest that being engaged in music activities may help a person connect with their life experiences and with other people, in turn, improving their quality of life (Lee et al., 2010)

In addition to how music affects health outcomes of older adults, there are also theories for how music may affect health outcomes within general hospital care. The theory of music, mood, and movement proposes that music alters mood, is a cue for movement, and makes physical activity more enjoyable, leading to improved health outcomes of weight, blood 
pressure, blood sugar, cardiovascular risk factor management, and improved quality of life (Murrock \& Higgins, 2009). The theory is based off how music has been shown to increase physical activity and improve health outcomes by taking into account the psychological and physiological responses of music. However, this theory is based off prior nursing interventions (Murrock \& Higgins, 2009). No additional research was found to support the proposed benefits of this theory. This theory may need to be tested first before determining its usefulness for improving health outcomes before any generalizations can be made for how it applies to older adults. Most recently, along the same lines of research dealing with quality of life and well-being from listening to music, researchers have tried to determine an individual's relationship between interest in music, health, and happiness within a single design (Rana, Akhtar, \& North, 2011). Researchers have achieved this by determining the interactions between 3 questionnaires: GHQ28 health questionnaire, MOH-45 measurement of happiness questionnaire, and IMQ interest in music questionnaire. After interpreting the data, the authors found a positive relationship between personal interests in listening to music, time spent listening to music, and each person's health and happiness scores, further supporting the benefits of music on health outcomes (Rana et al., 2011). Furthermore, data from this study was collected from 859 participants, far outweighing participant participation in previously described studies, which may decrease the uncertainty of self-reported data and lead to better generalizations for entire populations.

\section{Effects of Music on Anxiety}

Another well researched application of music is how it has been used as an intervention tool to affect stress and anxiety, especially in hospital patients. The State-Trait Anxiety Inventory (STAI) is used to measure anxiety in relation to music. White (1992) utilized this measurement to observe cardiac care patient's anxiety after listening to relaxing music. The results of the study 
showed that listening to music after surgery reduces anxiety. White (1992) additionally followed this study with a similarly designed study seven years later and found the same conclusions: state-trait anxiety levels decreased immediately after the music intervention (White, 1999). Adding to White's (1999) findings, a more recent review article provided further support to illustrate that music reduces anxiety in hospital patients (Evans, 2002). Evans (2002) analyzed a total of 12 randomized controlled trials, which evaluated the impact of music on anxiety. Final conclusions were consistent with White's (1999) findings; music also reduced anxiety during normal care delivery.

Music has also been found to reduce anxiety in more youthful populations. Labbe et al. (2007) utilized the STAI in a study of 56 college students who were exposed to classical music or self-selected relaxing music after exposure to a stressor. Participants once more demonstrated significant reductions in STAI regardless of the style of music. Conversely, Nilsson (2009) presented research that found no change in anxiety after listening to relaxing music. However, it is important to note that Nilsson (2009) used only investigator selected music. Subjects may have not preferred the type of music that was chosen. This choice may have been what caused the results to contradict what other researchers have found. Furthermore, a quiet rest environment has been shown to lower anxiety traits no-matter if music is present or not (White, 1992); further supporting that Nilsson's (2009) study may have included testing bias.

\section{$\underline{\text { Use of Music in Inpatient and Outpatient Cardiac Care Settings }}$}

For the purpose of the current study, it is vital to examine the effect of music found within cardiac care settings. Different hospitals may have different requirements to determine patient's status as inpatient or outpatient. Generally inpatient care can be described as the time when the doctor "admits" you to the hospital. Outpatient care is generally known as any time 
your doctor orders you, or you independently decide to participate in "observational" services that do not require you to occupy a bed, or stay overnight (different hospitals and clinics may have different standards for what is considered outpatient care).

The effects of music have been studied in both cardiac care settings. Inpatient care articles have typically studied music for its use as a stress relief or anxiety reducer (Elliott, 1994; White, 1992; Nilsson, 2009; Sendelbach et al., 2006; Voss et al., 2004; White, 1999). Patients who undergo cardiac surgery often times present high levels of anxiety. Research in this area has shown that music can also reduce anxiety pre and post different types of heart surgeries (Barnason et al., 1995; Hamel, 2001). In addition, music has also been shown to reduce stress after cardiac surgery (Nilsson, 2009), reduce pain, and improve mood during bed rest post open heart surgery (Barnason et al., 1995; Voss et al., 2004). No inpatient studies were found to complement music with exercise.

However, the effects of music have also been found in outpatient cardiac care settings. Three studies utilized music during exercise within a cardiac rehabilitation program. The earliest study found was conducted in 1995 (MacNay, 1995). MacNay (1995) utilized music with four adult male patients who exercised for 30 minutes on AirDyne bicycles to measure RPE and feeling status. Each subject had a chance to select their own preferred music. During exercise sessions with music, participants experienced lower RPE and higher positive feelings (MacNay, 1995). More recently, Murrock (2002) also observed patient's RPE and feeling scale in a study that involved 30 cardiac rehabilitation patients in a pretest/posttest design. In this study, patients experienced higher positive feelings (Murrock, 2002). However, no changes were seen in patients RPE between the music condition and no-music condition. The most recent article found within outpatient cardiac care programs was published by Emery et al. in 2003. Emery et al. 
(2003) asked 33 cardiac rehabilitation patients to complete two treadmill exercise sessions scheduled one week apart to observe HR, BP, anxiety, and cognitive function while listening to music. Before and after each exercise session participants completed a cognitive test of verbal fluency. The music session was consistent with improvements in verbal fluency, and the nomusic session was unassociated with cognitive change. No significant differences were found in any physiological variables.

While the main focus of using music in an outpatient CR program has been to reduce RPE and increase the feeling status of patients during exercise, the effects of music on RPE, BP, $\mathrm{HR}$, and feeling status in CR patients are controversial within the literature. A possible reason for this controversy may be due to music tempo, which can alter the effect that music has on these variables. No studies utilizing CR subjects were found to have included the effect of different tempos of music on HR, PRE, BP, or feeling status. The discrepancy in prior research may be due to the choices of the music tempo chosen by researchers. The upbeat tempo of certain stimulating music carries motivational factors, which contribute to improved performance or decreased RPE during exercise (Karageorghis \& Priest, 2012a). On the other hand, sedative music has only been associated with decreased RPE (Ghaderi et al., 2009; Hepler \& Kapke, 1996). Heart rate and BP have not been studied in CR programs in response do different tempos of music. In addition, no studies were found that observed music's effects on performance variables in this population. It would also be interesting to see if music increases performance during a standardized test in cardiac rehabilitation patients. 


\section{Summary}

Music has been associated with improving health outcomes and affecting humans in many ways during exercise. Due to the lack of research of music's effects in cardiac rehabilitation populations, this review has examined the effects of music across various populations. This review covered how music has been shown to affect physiological, psychological and psychophysical parameters. Lacking from the literature is a clear understanding of how music will impact individuals in an outpatient cardiac rehabilitation program. Key points from the review include (1) how different types and tempos of music affect exercise (2) the common physiological, psychological and psychophysical effects of music during exercise, and (3) how music has been found to affect individuals health parameters with and without an exercise component in inpatient and outpatient cardiac care programs. This information can now be taken and used as a road map to produce a study to best benefit cardiac rehabilitation patients during exercise.

It can now be inferred that if a researcher wants to increase performance, stimulating music would be the right choice versus sedative music. To counteract any negative feelings participants may encounter from music and increase the motivational qualities of a type of music, researchers can let subjects choose their preferred type of stimulating music. Furthermore, clinical implications for utilizing music in a cardiac rehabilitation program may include improved mood, increased performance, and decreased RPE. According to the literature, the main focal points of using music in an outpatient cardiac rehabilitation program has been to reduce RPE and increase the mood states of patients during exercise. The effects of music on RPE, HR, BP, and feeling states in cardiac patients are controversial thus far within the literature. This study aims to be a baseline for measuring these same variables. 


\section{CHAPTER III. METHODOLOGY}

The purpose of this study was to examine the effects of stimulating and sedative music on RPE, HR, BP, and feeling status during exercise in Phase III CR patients. All outcome measurements were investigated in response to each of the three music conditions. Prior to the experiment, subjects signed a written consent and gained permission from their primary care physician for participation. Procedures were also approved by the university's Institutional Review Board and the hospital's Review Board.

\section{Population Sample and Sampling Procedures}

A convenience sample of 22 male and female older adults age $64 \pm 8.0$ y currently enrolled in phase III CR at a local CR program completed the study. Subjects were recruited by the local CR staff. At the time of recruitment, subjects were provided a description of the criteria and given the option to participate if they met those criteria. Inclusion criteria required that subjects be willing to exercise on a treadmill at a speed of $3.0 \mathrm{mph}$ for 10 minutes. When observing HR and BP under any circumstances in rehabilitation, staff must also be aware that patients may be on medications that can alter HR and BP. The local CR program had documented this in their rehabilitation assessment prior to starting the program.

Phase III CR patients were recruited for two reasons. First, recruitment of phase III patients who are already familiar with treadmill exercise provides familiarity with exercise activity and our procedures (i.e. taking BP, HR, and RPE while exercising on a treadmill). To further reduce this effect, inclusion criteria also included prior acceptance into phase III program three weeks before the experiment to ensure they were well adapted to the equipment and procedures. Second, most patients in phase III programs have already participated in in phase II CR programs for an average of 12 weeks, allowing for physiological improvements post MI to have occurred. Phase II allows for rapid improvements in physiological and psychological 
measures over a short period of time. Excluding these phases will decrease the variability of time affecting our outcome measurements.

\section{$\underline{\text { Assessment Instruments }}$}

BP was monitored using Welch Allyn brand blood pressure cuffs (Welch Allyn Inc., Skaneateles Falls, NY) and a 3M Littman Quality Stethoscope (Owens and Minor Inc., Mounds View, MN). HR was monitored using a Series C M2601a telemetry transmitter. The Borg RPE scale was used to assess RPE (Appendix D). The Borg scale ranges from 6-20 with each unit increasing linearly with a qualitative effort description (e.g., 7/very very light, 9/very light, 11/fairly light, 13/somewhat hard, 15/hard, 17/very hard, and 19/very very hard). We assessed feeling status using a scale ranging from +5 to -5 with verbal anchors of +5 (very good), +3 (good), +1 (fairly good), 0 (neutral), -1 (fairly bad), -3 (bad), and -5 (very bad) (Appendix E). Subjects were also required to rate their enjoyment of each of the musical tracks via a Likert scale rating from (-2) really didn’t like, (-1) didn't like, (0) indifferent, (1) liked, and (2) really liked.

\section{$\underline{\text { Musical Selection }}$}

Stimulating music indicates fast-pace and uplifting music approximately 130-140 beats per minute (bpm). Sedative music represents slower-paced and more relaxing music approximately 90-100 bpm.

Musical tracks were selected by participants from an arrangement of over 50 popular stimulating and sedative oldies songs. Participants individually selected eight of their preferred songs, four from each tempo category. The highest marks for the top four sedative and top four stimulating tracks were chosen for use in this study. The four tracks for the sedative condition included: "She's Leaving Home” by The Beatles (94 bpm), "Unchained Melody" by The 
Righteous Brothers (90 bpm), "My Girl" by The Temptations (94 bpm), and "Imagine" by John Lennon (75 bpm). The four tracks chosen for the simulating condition included: "Oh, Pretty Woman" by Roy Orbison (128 bpm), "Roll Away the Stone" by Leon Russell (148 bpm), "House of the Rising Sun" by The Animals (128 bpm), and "It Don't Come Easy" by Ringo Starr (141 bpm). Subjects listened to music through Sony MDR-ZX100/WHI stereo headphones attached to a 4th generation iPod touch (Apple Inc., Cupertino, CA). Music tracks were played through Tempo Magic Pro downloaded from Apple Inc. application store.

\section{$\underline{\text { Procedures }}$}

Subjects arrived at the hospital at their preferred workout times. After ensuring the completion of all requirements and signing the informed consent form, they were connected to a telemetry transmitter to record heart rate and had 10 minutes of rest prior to the exercise. The researcher took the first recordings of all outcome measurements. At that time subjects were told that there would be another recording of those measurements half way through the exercise. Next, a music track of either stimulating music, sedative music, or a no-music control was provided to subjects through the iPod with attaching head phones. They then had the capability to change the volume of the track to their preference, but were instructed to not turn the track off. The exercise specialist then set the speed of the treadmill to $3.0 \mathrm{mph}$ and subjects performed the 10 min of treadmill exercise under one of the music conditions.

Subjects were randomized to differing music condition to avoid ordering effects. Data collection for each exercise session included RPE, BP, HR, and feeling status four times within the experiment. The music condition played consistently throughout the exercise and outcome measures were taken and recorded pre, at 5 minutes, at 10 minutes, and post exercise. The pre and post measurements were taken 10 minutes before and 10 minutes post exercise to ensure 
proper resting and recovery periods had occurred. During the treadmill exercise the exercise specialists would normally motivate and encourage the subjects. For the purposes of minimizing experimenter bias, minimal communication occurred only to record RPE, feeling status, and answering any health concerns subjects had during the exercise. All measurements were recorded and hand written onto a printed excel sheet. After completion of the first experimental session participants were scheduled to return for two additional sessions. Each exercise session was separated by 48 hours to ensure adequate recovery and a reasonable washout period for the crossover design.

A crossover design was used to guide data collection. The manipulated independent variable was music condition (sedative, stimulating, and non-music control). The dependent variables were RPE, BP, HR, and feeling status with each represented by four repeated measures over time. Separate ANOVAs assessed differences in the four dependent variables over time. Rough sample size calculations based on paired t-tests were used to obtain sample size estimates (SAS Institute Inc., 2011). A-priori analysis suggested that a sample size of 12 would result in power $=.8$ for within subjects effects. 


\section{CHAPTER IV. ARTICLE}

\section{$\underline{\text { Introduction }}$}

Cardiac Rehabilitation (CR) specialists may be able to expand upon the benefits of phase III CR programs by incorporating music into their patient's exercise routine. Current research suggests listening to music may enhance physical activity during sport and exercise (Karageorghis \& Priest, 2012a, 2012b; Karageorghis \& Terry, 1997). A large portion of current research focuses on the effects of music on exercise performance within high school to collegeaged populations. Few studies exist observing the impact of music on exercise-related variables within CR populations (Emery et al., 2003; Murrock, 2002; MacNay, 1995). The clinical setting warrants further examination of more clinical outcomes of listening to music during exercise (blood pressure [BP], heart rate [HR], rating of perceived exertion [RPE], and feeling status).

Previous clinical trials have attempted to use music to reduce RPE and increase feeling status during exercise in CR populations. The rationale is that compliance with an exercise program depends on the degree of pleasure associated with the exercise. The more pleasure perceived, the more likely the exercise will be repeated to promote exercise compliance (Murrock, 2002). Therefore, improvement in feeling status during exercise should also enhance compliance to the exercise program. Similarly, if a patient's RPE is lowered while exercising to music, exercise may be done more effortlessly, which can also lead to physiological improvements (Murrock, 2002).

There is a lack of consensus concerning the effects of music on RPE, HR, BP, and feeling status during exercise in $\mathrm{CR}$ patients. One factor that may explain these varied results is the tempo of music. To our knowledge, no clinical study has examined the effect of music tempo on those variables during exercise. 
The purpose of this study is to examine the effects of stimulating and sedative music on RPE, HR, BP, and feeling status during exercise in Phase III CR patients.

\section{$\underline{\text { Methods }}$}

A convenience sample of 22 male and female older adults age $64 \pm 8.0$ y currently enrolled in phase III CR at a local CR program completed the study (See Table 1). During recruitment, subjects were provided a description of the inclusion criteria and given the option to participate. Inclusion criteria required that subjects be willing to exercise on a treadmill at a speed of $3.0 \mathrm{mph}$ for 10 minutes. Prior to the experiment, subjects signed a written consent and gained permission from their primary care physician for participation. Procedures were approved by the university's Institutional Review Board and the hospital's Review Board.

Table 1. Demographics

\begin{tabular}{|c|c|c|c|c|}
\hline Gender & $\begin{array}{l}\text { Avg. Age } \\
\text { Years }\end{array}$ & Race & $\begin{array}{l}\text { Mean Resting HR } \\
\text { bpm }\end{array}$ & $\begin{array}{l}\text { Mean Resting BP } \\
\mathrm{mmHg}\end{array}$ \\
\hline Male 17 & $66.3 \pm 7.1$ & Caucasian & $68.2 \pm 9.4$ & $114 \pm 10.6 / 68 \pm 6.5$ \\
\hline Female 5 & $56.6 \pm 6.6$ & Caucasian & $74.8 \pm 5.9$ & $117 \pm 8.6 / 71 \pm 6.4$ \\
\hline
\end{tabular}

\section{Assessment Instruments}

BP was monitored using Welch Allyn brand blood pressure cuffs (Welch Allyn Inc., Skaneateles Falls, NY) and a 3M Littman Quality Stethoscope (Owens and Minor Inc., Mounds View, MN). HR was monitored using a Series C M2601a telemetry transmitter. The Borg RPE scale was used to assess RPE (Borg, 1982). The Borg scale ranges from 6-20 with each unit increasing linearly with a qualitative effort description (e.g., 7/very very light, 9/very light, 11/light, 13/somewhat hard, 15/hard, 17/very hard, and 19/very very hard). We assessed feeling status using a scale ranging from +5 to -5 with verbal anchors of +5 (very good), +3 (good), +1 (fairly good), 0 (neutral), -1 (fairly bad), -3 (bad), and -5 (very bad) (Rejeski, 1985). Subjects 
were also required to rate their enjoyment of each of the musical tracks via a Likert scale rating from (-2) really didn’t like, (-1) didn’t like, (0) indifferent, (1) liked, and (2) really liked.

\section{Musical Selection}

Stimulating music indicates fast-pace and uplifting music approximately 130-140 beats per minute (bpm). Sedative music represents slower-paced and more relaxing music approximately $90-100 \mathrm{bpm}$.

Musical tracks were selected by participants from an arrangement of over 50 popular stimulating and sedative oldies songs. Participants individually selected eight of their preferred songs, four from each tempo category. The highest marks for the top four sedative and top four stimulating tracks were chosen. The four tracks for the sedative condition included: "She's Leaving Home” by The Beatles (94 bpm), "Unchained Melody” by The Righteous Brothers (90 bpm), "My Girl” by The Temptations (94 bpm), and "Imagine” by John Lennon (75 bpm). The four tracks chosen for the simulating condition included: “Oh, Pretty Woman” by Roy Orbison (128 bpm), "Roll Away the Stone" by Leon Russell (148 bpm), "House of the Rising Sun" by The Animals (128 bpm), and “It Don't Come Easy” by Ringo Starr (141 bpm). Subjects listened to music through Sony MDR-ZX100/WHI stereo headphones attached to a 4th generation iPod touch (Apple Inc., Cupertino, CA). Music tracks were played through Tempo Magic Pro downloaded from Apple Inc. application store.

\section{Procedures and Statistical Analysis}

Participants completed the exercise protocol on three separate visits to the clinic. Music condition (sedative, stimulating, and control) was randomly assigned to avoid ordering effects. Each participant's exercise session consisted of a 10 minute treadmill exercise at $3.0 \mathrm{mph}$, where they were exposed to the music condition throughout the entire protocol. Variables were 
collected 10 minutes before and after exercise, and at minutes 5 and 10 during exercise. Pre and post measurements were taken 10 minutes before and 10 minutes after exercise to ensure proper resting and recovery periods occurred. Each exercise session was separated by 48 hours to ensure adequate recovery and a reasonable washout period for the crossover design.

A crossover design was used to guide data collection. The manipulated independent variable was music condition (sedative, stimulating, and non-music control). The dependent variables were RPE, BP, HR, and feeling status with each represented by four repeated measures over time. Separate ANOVAs assessed differences in the four dependent variables over time. Rough sample size calculations based on paired t-tests were used to obtain sample size estimates (SAS Institute Inc., 2011). A-priori analysis suggested that a sample size of 12 would result in power $=.8$ for within subjects effects.

\section{$\underline{\text { Results }}$}

Data analysis was done using a crossover design for each dependent measure at each time period. This approach treated repeated measures within each session by running each time within a patient session as a separate dependent variable. In other words, there was a crossover design on each exercise related variable of interest (HR, BP, RPE, and feeling status) for the pre, 5 minute, 10 minute, and post data alone. The means and standard deviations are listed in Table 2. ANOVA analysis revealed significant differences in HR, RPE and feeling status exercise related variables during exercise at both 5 minute, and 10 minute time points. Systolic and diastolic blood pressures were the only variables where significance was not found at 5 minute $(p=.3040)$ or 10 minute $(p=.8512)$ measurements. 
Table 2. Means with Standard Deviations

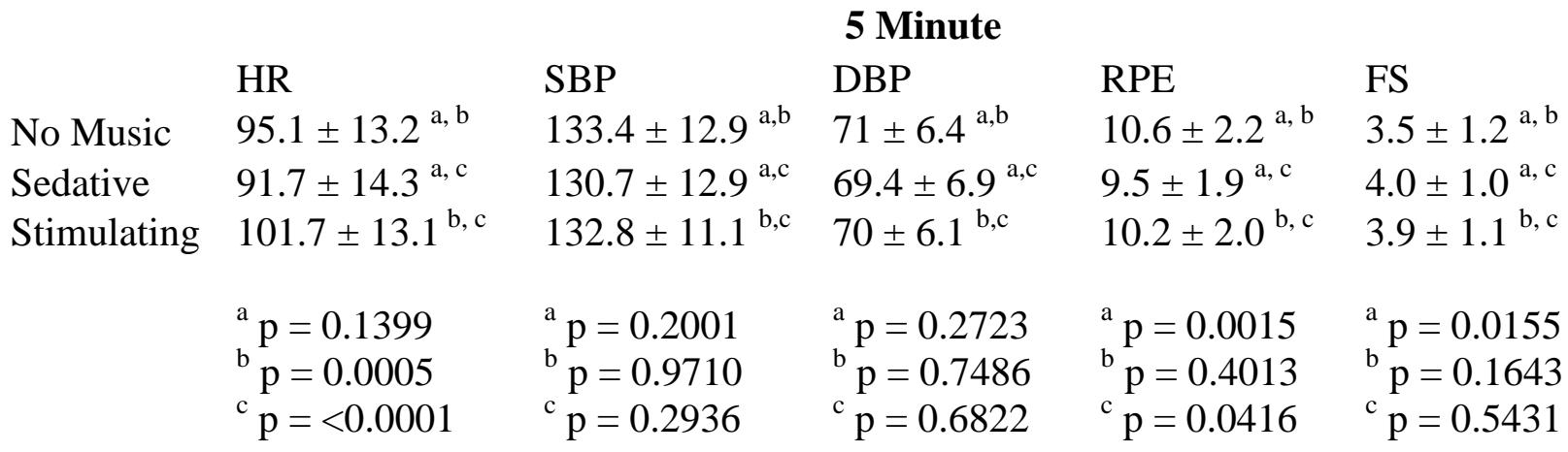

\section{Minute}

No Music $\quad 96.9 \pm 14.1^{\mathrm{a}, \mathrm{b}} \quad 134.7 \pm 11.6^{\mathrm{a}, \mathrm{b}} \quad 70.8 \pm 6.1^{\mathrm{a}, \mathrm{b}}$

$11.3 \pm 2.5^{\mathrm{a}, \mathrm{b}} \quad 3.6 \pm 1.1^{\mathrm{a}, \mathrm{b}}$

Sedative $\quad 92.5 \pm 14.2^{\mathrm{a}, \mathrm{c}}$

$133.6 \pm 11.2^{\mathrm{a}, \mathrm{c}} \quad 70.1 \pm 7.0^{\mathrm{a}, \mathrm{c}}$

$9.9 \pm 2.0^{\mathrm{a}, \mathrm{c}}$

$4.0 \pm 1.0^{\mathrm{a}, \mathrm{c}}$

Stimulating $102.5 \pm 12.5^{\mathrm{b}, \mathrm{c}}$

$135.9 \pm 12.3^{\mathrm{b}, \mathrm{c}} 70.4 \pm 7.0^{\mathrm{b}, \mathrm{c}}$

$10.5 \pm 1.9^{b, c}$

$3.9 \pm 1.1^{\mathrm{b}, \mathrm{c}}$

$$
\begin{aligned}
& { }^{\mathrm{a}} \mathrm{p}=0.0516 \\
& { }_{\mathrm{b}} \mathrm{p}=0.0056 \\
& { }_{\mathrm{c}} \mathrm{p}=<0.0001
\end{aligned}
$$

${ }^{\mathrm{a}} \mathrm{p}=0.8082$

${ }^{\mathrm{b}} \mathrm{p}=0.6144$
${ }^{\mathrm{a}} \mathrm{p}=0.8468$
${ }^{\mathrm{b}} \mathrm{p}=0.9874$
${ }^{c} \mathrm{p}=0.9162$

${ }^{\mathrm{a}} \mathrm{p}=0.0002$

${ }^{\mathrm{b}} \mathrm{p}=0.0709$

${ }^{\mathrm{a}} \mathrm{p}=0.0468$

${ }^{c} \mathrm{p}=0.2702$

${ }^{\mathrm{b}} \mathrm{p}=0.3874$

${ }^{c} \mathrm{p}=0.4995$

${ }^{a} p=p$-value when comparing ${ }^{a}$ to ${ }^{a}$ (No Music vs Sedative)

${ }_{\mathrm{p}}^{\mathrm{p}}=\mathrm{p}$-value when comparing ${ }^{\mathrm{b}}$ to ${ }^{\mathrm{b}}$ (No Music vs Stimulating)

${ }^{c} p=p$-value when comparing ${ }^{c}$ to ${ }^{c}$ (Sedative vs Stimulating) 
Figure 1 illustrates the mean (plus symbol), median (horizontal line in body of shaded box), and upper and lower quartiles (top and bottom of shaded box) for each music condition within each time period measured during an exercise session. The whiskers illustrate variation up to a maximum of one interquartile range (IQR) from the upper and lower quartiles. Any points more than one IQR from the upper or lower quartiles are shown with a separate symbol.

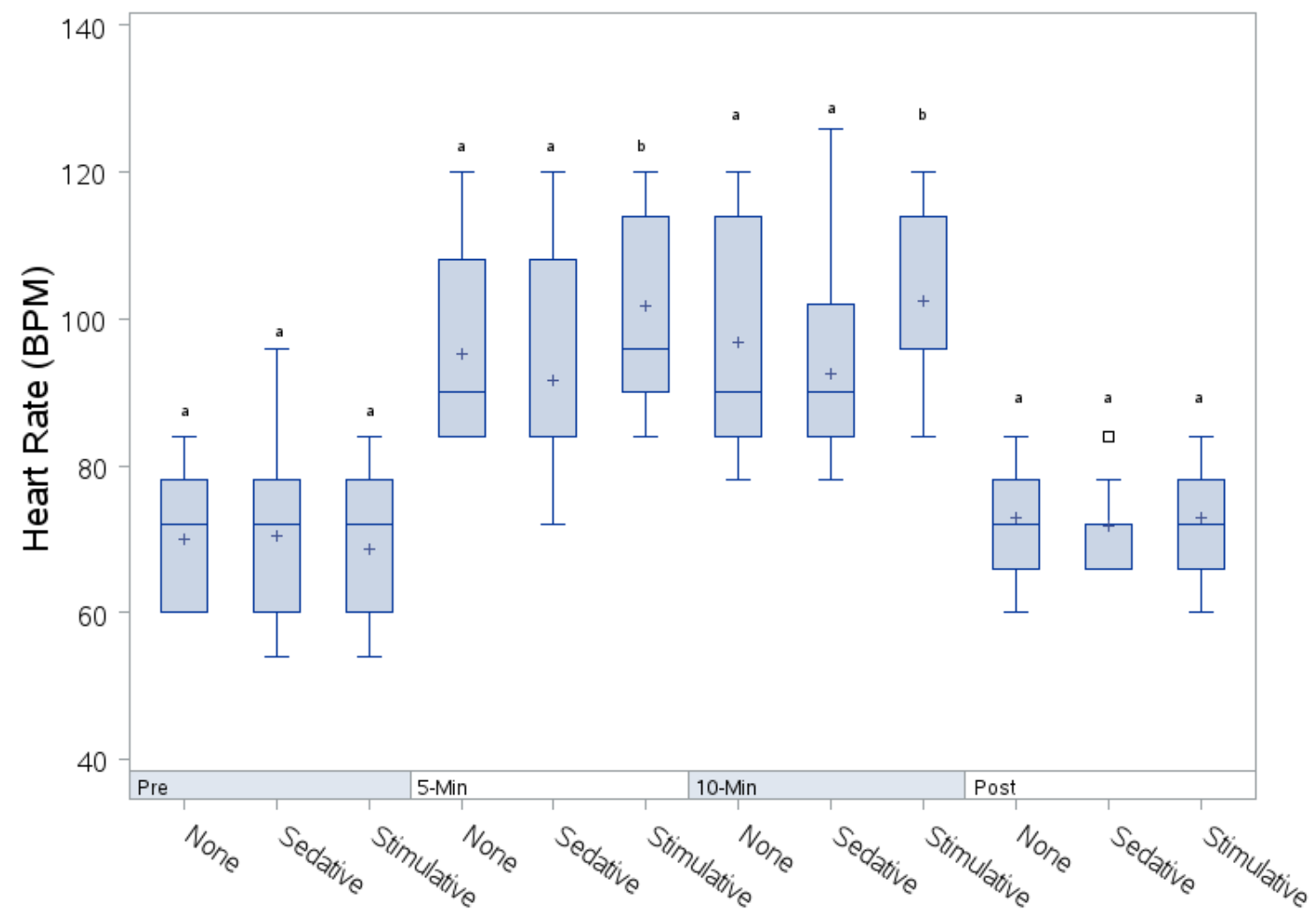

Figure 1. Effect of Music on HR.

${ }^{\mathrm{b}}=$ statistically significant when compared to ${ }^{\mathrm{a}}$ at $\mathrm{p}<.05$

${ }^{\mathrm{a}}=$ not statistically significant when compared to ${ }^{\mathrm{a}}$

Significant differences for HR were observed during both the 5 minute $F(2,40)=19.53$, $p<.0001$, and 10 minute $F(2,40)=16.51, p<.0001$ analysis (Table 2 ). Tukey-Kramer post-hoc analysis of 5 minutes showed that HR under the stimulating music condition was higher than 
both the no music (adjusted $p=.0005$ ), and sedative music conditions (adjusted $p<.0001$ ). Very similar adjusted $\mathrm{p}$-values were observed at 10 minutes $(p=.0056, p<.0001$ respectively).

Figure 2 notes changes in RPE. Attributes of the boxes are as described in Figure 1. Note that there was no Pre or Post data collected so that portion of the figure is blank.

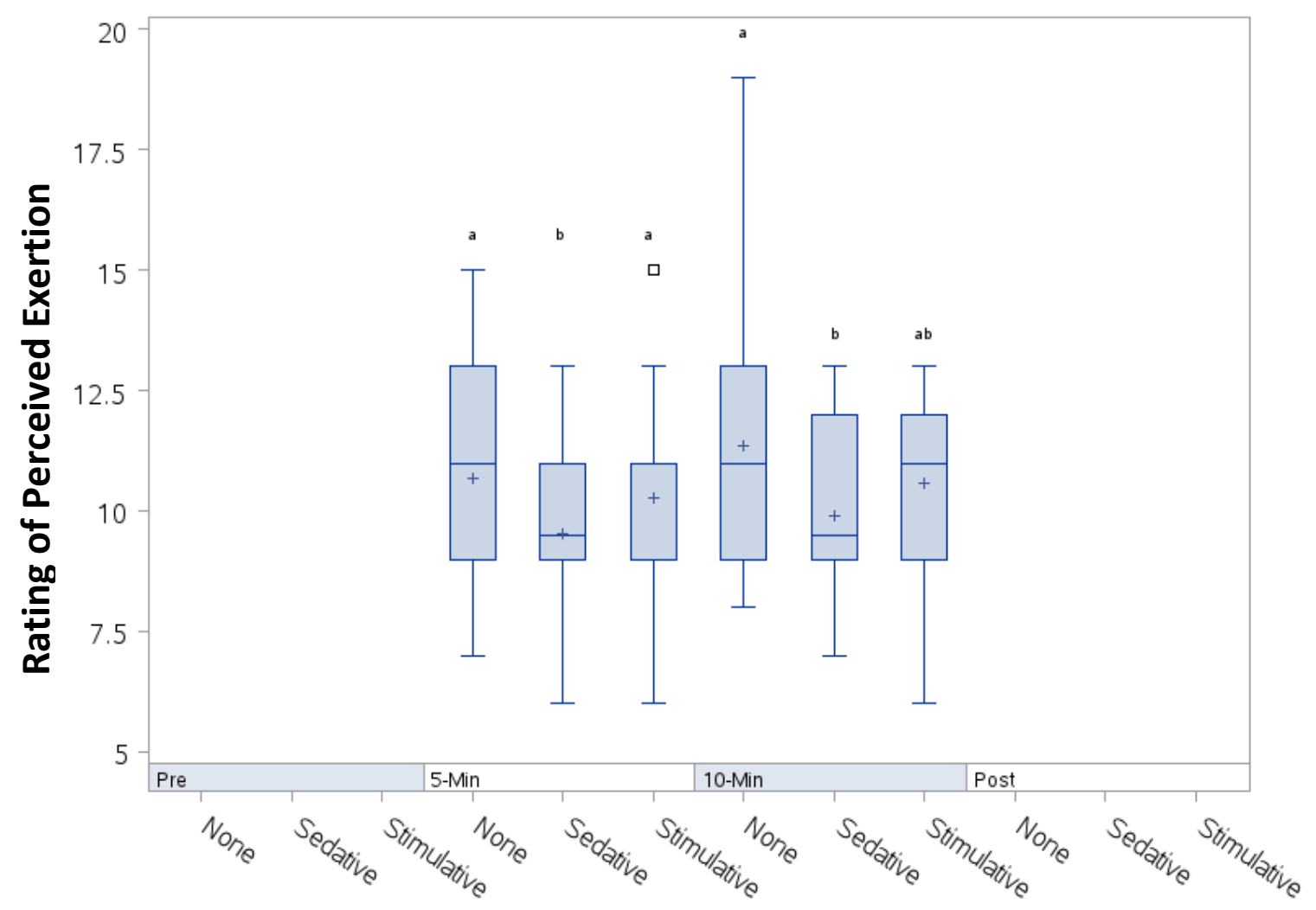

Figure 2. Effect of Music on RPE.

${ }^{\mathrm{b}}=$ statistically significant at $\mathrm{p}<.05$ from $^{\mathrm{a}}$

${ }^{\mathrm{a}}=$ not statistically significant when compared to ${ }^{\mathrm{a}}$

${ }^{\mathrm{ab}}=$ statistically significant at $\mathrm{p}<.05$ from $^{\mathrm{a}}$ and ${ }^{\mathrm{b}}$

RPE measurements were only recorded during exercise as they are a measurement of perceived exertion and there is no exertion at rest. Differences were observed for RPE during the 5 minute $F(2,40)=7.33, p=.0001$, and 10 minute recordings $F(2,40)=9.74, p=.0004$ (Table 2). Tukey-Kramer post-hoc analysis for the adjusted p-value at 5 minutes showed that RPE was 
higher under the no music condition than sedative $(p=.0015)$, and that the stimulating music condition was higher than sedative $(p=.0466)$. Sedative music presented the lowest RPE recordings with stimulating music being slightly higher, and no music presenting the highest RPE recordings (Figure 2). The same sequence of results held true for the 10 minute recordings as well ( $p=.0004)$. However, at 10 minutes the adjusted $p$-values revealed that only the no music condition was significantly higher than the sedative condition $(p=.0002)$.

The ANOVA revealed significant differences for feeling status during the 5 minute $F(2,40)=4.37, p=.0192$ and post recordings $F(2,40)=4.49, p=.0174$, but not initially at 10 minutes $F(2,40)=3.04, p=.0590$ (Table 2). However, the Tukey-Kramer analysis of the adjusted p-value at 10 minutes revealed that the sedative music condition was higher than no music $(p=.0468)$ which correlates with the other time points. 


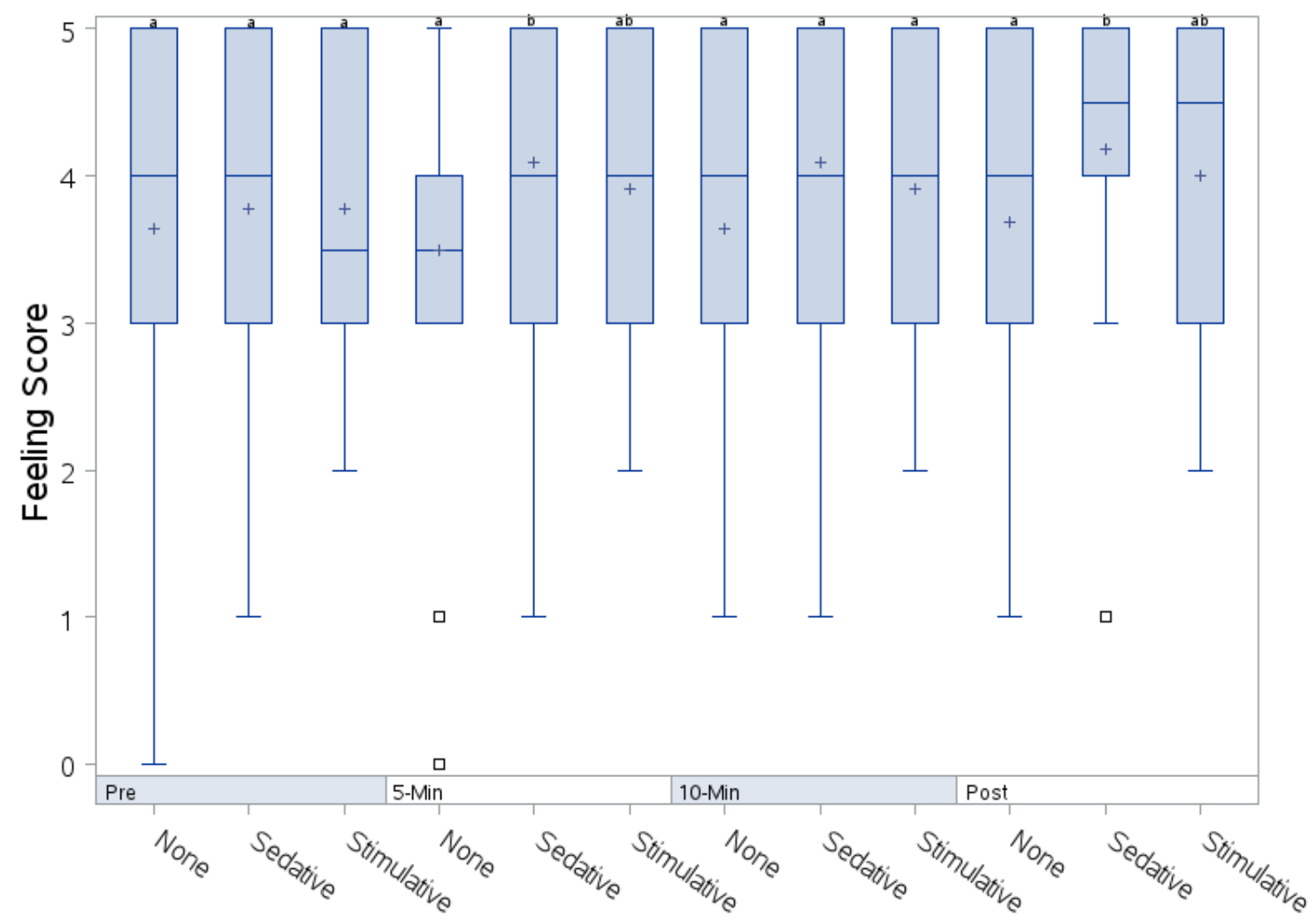

Figure 3. Effect of Music on Feeling Status.

${ }^{\mathrm{b}}=$ statistically significant at $\mathrm{p}<.05$ from $^{\mathrm{a}}$

${ }^{a}=$ not statistically significant when compared to ${ }^{\mathrm{a}}$

${ }^{\mathrm{ab}}=$ statistically significant at $\mathrm{p}<.05$ from $^{\mathrm{a}}$ and $^{\mathrm{b}}$

The adjusted p-value at 5 minutes showed higher feeling status scores under the sedative music condition compared with no music ( $p=.0155)$. Participants also continued to experience higher feeling status scores from sedative music over no music after the exercise was completed ( $p=.0131)$. At 5 minutes and 10 minutes there were no differences between no music and stimulating, or sedative and stimulating conditions. 


\section{$\underline{\text { Discussion }}$}

\section{Heart Rate}

At both the 5 minute and 10 minute time observations we detected an increase in HR in response to stimulating music, and a decrease in HR in response to sedative music $(\mathrm{p}<.001)$. By comparison, researchers report that listening to stimulating music at rest results in increased HR and respiratory rate when participants get the chance to select their own piece of stimulating music (Lingham \& Theorell, 2009). This response could be due to a feeling of joy, and elated mood and energy while listening to stimulating music (Lingham \& Theorell, 2009). In the same study, when individuals select their own piece of sedative music, it induced feelings of calmness and relaxation which lowered HR. Previous findings are consistent with our results of the effect of music on HR with the addition of exercise (Figure 1).

On the contrary, heart-rate, much like stroke volume and cardiac output, increases in a linear fashion with increases in the intensity of aerobic exercise. No matter if music is present during exercise we would expect an increase in HR with the rise in exercise intensity. Yamashita et al. (2006) came to the conclusion that the influence of music on HR was minimal and not significant, when they had 8 healthy adult males exercise to their favorite musical piece at $40 \%$ and $60 \%$ VO2 max. In fact, differences in autonomic activity were concluded to be affected only by the exercise intensity and not by music (Yamashita et al., 2006). However, the researchers used tempos of music ranging from 98-162 bpm. In our population of older adults, and during moderate exercise intensities, we now know that the tempo of music effects HR during exercise. It is possible that even at high intensities, such as $60 \% \mathrm{VO} 2 \mathrm{max}$, that the tempo of music should be of interest when analyzing HR data, as the outcome may vary with respect to sedative and stimulating music conditions. The only practical approach may be to standardize the intensity of 
exercise as we have done. During standardized exercise stimulating music increases HR, and sedative music decreases HR when analyzing with a within subjects control.

Although this may be true, observations of CR patients show that certain medications such as beta blockers put a ceiling on subject's HR. Fifteen of the 22 subjects were found to have been on a beta blocker. Nevertheless, we still observed significance at moderate exercise intensities, and have presented other possible pathways to increase or decrease HR without altering the exercise routine. It is not clear how our results could be generalized over populations and at higher exercise intensities.

\section{Blood Pressure}

Emery et al. (2003) had CR subjects complete two 10 minute treadmill exercise sessions to examine the effects of sedative music on BP. During one of the exercise sessions participants listened to sedative music, and during the other participants listened to a blank tape. Emery et al. (2003) concluded that there were no significant differences in blood pressure between the two groups, consistent with our findings for both music conditions while exercising. We observed no differences at 5 minutes or 10 minutes for systolic $(p=.1777, p=.2975)$ or diastolic $(p=.3040$, $p=.8512$ ) measurements. During exercise, music appears to have no effect on the nervous and endocrine systems that regulate blood pressure. As with HR however, BP medications could be limiting factors while observing CR patients. It is not clear how the observed effects of our study may generalize to healthy older adults outside of our setting.

Consequently, we believe that if researchers want to best utilize sedative music in a nonexercise setting, then it would be the right choice to reduce anxiety and stress, and to improve mood as found in other research (White, 1992). There appears to be no indication of immediate 
pre, during, or post blood pressure reducing effects of either sedative or stimulating music during exercise in this population.

\section{Feeling Status}

The feeling status scale is a measure for how a person feels during exercise (Hardy \& Rejeski, 1989). Hardy and Rejeski (1989), suggest that although RPE represents what an individual feels during exercise, it does not reflect how a person feels. Rejeski's (1985) parallel information processing model suggests that sensory and emotional information are processed in parallel through focal awareness. Therefore, our perception of effort (sensory information) or apprehension of exercise (affective information) form the object of attention and determine how we feel during exercise (Rejeski, 1985). They further propose that RPE is insensitive to whether the reported exertion is based on informational or emotional cues, and relies on physiological input of how hard a person thinks they're working. For example, two individuals may report an RPE score of 15 . One may be under a considerable amount of physiological and emotional distress whereas the other may be feeling physiologically strained but feels no emotional distress. The feeling scale takes this into account and relies on a more emotional state of feeling.

We previously stated that an improvement in feeling status during exercise should also enhance compliance to the exercise program. It is especially important in a cardiac rehabilitation program to know how a patient feels in accordance with how hard a patient thinks they are working due to possible clinical complications during exercise. In respect to that, it is of utmost importance that the participants experience a sense of pleasure from the music. One way to be sure of this was to employ a Likert scale rating of how participants felt about the music. After each exercise session subjects rated how much they liked the music condition on a scale of really liked $(+2)$ down to really didn't like (-2). Out of 22 subjects, 19 really liked (+2) all music 
conditions and only 3 subjects presented ratings of liked (+1), 2 were for sedative and 1 was for stimulating music. No participants presented ratings from neutral (0) to really didn't like (-2). We have assumed that the Likert scale plus our gathering methods for musical selection represented music that was preferred by patients.

Evidently, sedative music produced the highest feeling status scores at 5 minutes $(p=.0155)$ and 10 minutes $(\mathrm{p}=.0468)$ when compared to no music. Stimulating music also increased feeling status scores but not significantly at $5(p=.1643)$ or 10 minutes $(p=.3874)$ when compared to no music. Future research will need to be done to determine why sedative music claimed the best outcomes on feeling status.

\section{Rating of Perceived Exertion}

Researchers have found that music plays a key role in lowering RPE during exercise: (Boutcher \& Trenske, 1990; Mohammadzadeh, Tartibiyan, \& Ahmadi, 2008a; Yamashita, Iwai, Akimoto, Sugawara, \& Kono, 2006). Each researcher has suggested that music does lower RPE, but only significantly lowers RPE at low to moderate intensities. An explanation for this effect could be that participants may only react to the effects of music as a passive distractor during treadmill exercise (Bharani, Sahu, \& Mathew, 2004). Once the exerciser reaches high workloads or high intensities the individual may not pay attention to the music anymore and focus their attention back towards the task, therefore eliminating effects of the music on RPE. With this information, researchers could expect that when music is used at low intensities as a distractor, it may lower RPE, but once the physiological effects of the exercise reaches a higher intensity (which is variable to each individual) the distractor may not be beneficial anymore.

In addition to using lower exercise intensities, for best results in lowering RPE during exercise, we have also observed that it is vital that the tempo of music be sedative. This 
correlates with researchers who found that the use of music during exercise sessions could decrease RPE when the style of music was easy listening/slow (Copeland \& Franks, 1991).

Consequently in the current study, actual RPE means varied between $9.5 \pm 1.9$ (very light) under the sedative music condition and $11.3 \pm 2.5$ (light) under the no music condition. RPE scores that are reported this low during exercise would call for a change to the participants exercise routine, allowing them to exercise at a higher intensity for that day. At our local facility the rule of thumb is to have patients exercise between 11 (light) and 15 (hard) on the RPE scale. While standardizing the exercise, we now know that RPE decreases under sedative music conditions more than both stimulating and no music conditions (Table 2). However, this may only be applicable in the case that the exerciser was $>15$ as to bring them back down to $\leq 15$ without changing the exercise routine. In that case, sedative music may be the answer. This assumption should be tested at higher intensities.

\section{Conclusions and Recommendations}

The purpose of this study was to examine the effects of stimulating and sedative music on RPE, HR, BP, and feeling status during exercise in Phase III CR patients. Due to the lack of consensus concerning how sedative and stimulating music affects these variables, the results would then provide a more straight forward analysis in hopes to discern the two music conditions.

Previous clinical trials have attempted to use music to reduce RPE and increase feeling status during exercise in CR populations, but it was unclear as to whether music tempo was a viable component for the lack of consensus. While standardizing the exercise, we now see that sedative music is the best choice to observe decreases RPE, increases in feeling status, and decreases HR. Stimulating music would only be the correct choice to observe increases in HR, 
and does not have as much of a beneficial effect on RPE and feeling status as sedative music. There were no significant effects of either type of music on BP.

To our knowledge, no clinical study has examined the effect of music tempo on these variables during exercise in CR patients. The intensity and duration of the exercise play into how music affects the exerciser, as well as controlling for the use of medications that may alter BP and HR. To observe changes in RPE and feeling status researchers have suggested that these variables may only be affected at lower intensities; which we have observed in the current study. We have also observed that the choice of music must be sedative/slow paced for optimal results. It is unknown as to whether these conclusions will hold true at higher intensities of exercise. It is clinically meaningful that CR programs be aimed at improving heart health measures. To recommend that further research be directed to lower intensities of exercise for experiencing more positive effects of music, may be to oppose the recommendations of phase III CR programs for those who seek gradual improvements in exercise intensity, MET level, etc.

In this phase of $\mathrm{CR}$ we suggest that future research be directed at testing patients while they exercise on different pieces of equipment that are preferred by the patients (stationary bicycle, EFX, nu-step, etc.). Alternative pieces of equipment may induce different exercise intensities as well as feeling of pleasure that may also affect RPE and feeling status. Future research should also look toward utilizing background music rather than I-pod and headphones. The headphones have the added benefit of blocking the hospital environment while listening to music. The extra auditory stimuli in that environment should be accompanied with the music for an optimal natural setting observation. Furthermore, playing the music throughout the entire protocol would provide added insight as to how music affects the exerciser at rest before and after the exercise. 


\section{$\underline{\text { References }}$}

Bharani, A., Sahu, A., \& Mathew, V. (2004). Effect of passive distraction on treadmill exercise test performance in healthy males using music. International Journal of Cardiology, 97(2), 305-306.

Borg, G. A. V. (1982). Psychophysical bases of perceived exertion. Medicine \& Science in Sports \& Exercise, 14(5), 377-381.

Boutcher, S. H., \& Trenske, M. (1990). The effects of sensory deprivation and music on perceived exertion and affect during exercise. Journal of Sport \& Exercise Psychology, 12(2), 167-176.

Copeland, B.L., \& Franks, B.D. (1991). Effects of types and intensities of backround music on treadmil endurance. Journal of Sports Medicine and Physical Fitness, 31(1), 100-103.

Emery, C. F., Hsiao, E. T., Hill, S. M., \& Frid, D. J. (2003). Short-term effects of exercise and music on cognitive performance among participants in a cardiac rehabilitation program. Heart \& Lung, 32(6), 368-373.

Hardy, C. J., \& Rejeski, W. J. (1989). Not what, but how one feels: The measurement of affect during exercise. Journal of Sport \& Exercise Psychology, 11(3), 304-317.

Karageorghis, C. I., \& Priest, D.-L. (2012a). Music in the exercise domain: A review and synthesis (Part I). International Review of Sport \& Exercise Psychology, 5(1), 44-66.

Karageorghis, C. I., \& Priest, D.-L. (2012b). Music in the exercise domain: A review and synthesis (Part II). International Review of Sport \& Exercise Psychology, 5(1), 67-84.

Karageorghis, C. I., \& Terry, P. C. (1997). The psychophysical effects of music in sport and exercise: A review. Journal of Sport Behavior, 20(1), 54. 
Lingham, J., \& Theorell, T. (2009). Self-selected "favourite" stimulative and sedative music listening -- how does familiar and preferred music listening affect the body? Nordic Journal of Music Therapy, 18(2), 150-166.

MacNay. (1995). The influence of preferred music on the perceived exertion, mood, and time estimation scores of patients participating in a cardiac rehabilitation program. Music Therapy Perspectives, 13, 91-96

Mohammadzadeh, H., Tartibiyan, B., \& Ahmadi, A. (2008). The effects of music on the perceived exertion rate and performance of trained and untrained individuals during progressive exercise. Facta Universitatis: Series Physical Education \& Sport, 6(1), 6774.

Murrock, C. J. (2002). The effects of music on the rate of perceived exertion and general mood among coronary artery bypass graft patients enrolled in cardiac rehabilitation phase II. Rehabilitation Nursing, 27(6), 227-231.

Rejeski, W. J. (1985). Perceived exertion: An active or passive process? Journal of Sport Psychology, 7(4), 371-378.

SAS Institute Inc. 2011. SAS/STAT® 9.3 User’s Guide. Cary, NC: SAS Institute Inc.

White, J. M. (1992). Music Therapy: An intervention to reduce anxiety in the myocardial infarction patient. Clinical Nurse Specialist, 6(2), 58-63.

Yamashita, S., Iwai, K., Akimoto, T., Sugawara, J., \& Kono, I. (2006). Effects of music during exercise on RPE, heart rate and the autonomic nervous system. Journal of Sports Medicine \& Physical Fitness, 46(3), 425-430. 


\section{CHAPTER V. SUMMARY, CONCLUSIONS, AND RECOMMENDATIONS FOR FURTHER}

\section{STUDY}

The purpose of this study was to examine the effects of stimulating and sedative music on RPE, HR, BP, and feeling status during exercise in Phase III CR patients. Due to the lack of consensus concerning how sedative and stimulating music affects these variables, the results would then provide a more straight forward analysis in hopes to discern the two music conditions.

Previous clinical trials have attempted to use music to reduce RPE and increase feeling status during exercise in $\mathrm{CR}$ populations, but it was unclear as to whether music tempo was a viable component for the lack of consensus. While standardizing the exercise, we now see that sedative music is the best choice to observe decreases RPE, increases in feeling status, and decreases HR. Stimulating music would only be the correct choice to observe increases in HR, and does not have as much of a beneficial effect on RPE and feeling status as sedative music. There is no significant effect of either type of music on BP.

To our knowledge, no clinical study has examined the effect of music tempo on these variables during exercise in CR patients. The intensity and duration of the exercise play into how music affects the exerciser, as well as controlling for the use of medications that may alter BP and HR. To observe changes in RPE and feeling status researchers have suggested that these variables may only be affected at lower intensities; which we have observed in the current study. We have also observed that the choice of music must be sedative/slow paced for optimal results. It is unknown as to whether these conclusions will hold true at higher intensities of exercise. It is clinically meaningful that $\mathrm{CR}$ programs be aimed at improving heart health measures. To recommend that further research be directed to lower intensities of exercise for experiencing 
more positive effects of music, may be to oppose the recommendations of phase III CR programs for those who seek gradual improvements in exercise intensity, MET level, etc.

In this phase of CR we suggest that future research be directed at testing patients while they exercise on different pieces of equipment that are preferred by the patients (stationary bicycle, EFX, nu-step, etc.). Alternative pieces of equipment may induce different exercise intensities as well as feeling of pleasure that may also affect RPE and feeling status. Future research should also look toward utilizing background music rather than I-pod and headphones. The headphones have the added benefit of blocking the hospital environment while listening to music. The extra auditory stimuli in that environment should be accompanied with the music for an optimal natural setting observation. Furthermore, playing the music throughout the entire protocol would provide added insight as to how music affects the exerciser at rest before and after the exercise. 


\section{REFERENCES}

Anshel, M. H., \& Marisi, D. Q. (1978). Effect of music and rhythm on physical performance. Research Quarterly, 49(2), 109-113.

Barnason, S., Zimmerman, L., \& Nieveen, J. (1995). The effects of music interventions on anxiety in the patient after coronary artery bypass grafting. Heart \& Lung, 24(2), 124132.

Barwood, M. J., Weston, N. J., Thelwell, R., \& Page, J. (2009). A motivational music and video intervention improves high-intensity exercise performance. Journal of Sports Science \& Medicine, 8(3), 435-442.

Bauldoff, G. S., Hoffman, L. A., Zullo, T. G., \& Sciurba, F. C. (2002). Exercise maintenance following pulmonary rehabilitation. CHEST, 122(3), 948.

Bharani, A., Sahu, A., \& Mathew, V. (2004). Effect of passive distraction on treadmill exercise test performance in healthy males using music. International Journal of Cardiology, 97(2), 305-306.

Borg, G. A. V. (1982). Psychophysical bases of perceived exertion. Medicine \& Science in Sports \& Exercise, 14(5), 377-381.

Boutcher, S. H., \& Trenske, M. (1990). The effects of sensory deprivation and music on perceived exertion and affect during exercise. Journal of Sport \& Exercise Psychology, 12(2), 167-176.

Brownley, K. A., McMurray, R. G., \& Hackney, A. C. (1995). Effects of music on physiological and affective responses to graded treadmill exercise in trained and untrained runners. International Journal Of Psychophysiology, 19(3), 193-201. 
Byers, J. F., \& Smyth, K. A. (1997). Effect of a music intervention on noise annoyance, heart rate, and blood pressure in cardiac surgery patients. American Journal Of Critical Care, 6(3), 183-191.

Chang, H.-K., Peng, T.-C., Wang, J.-H., \& Lai, H.-L. (2011). Psychophysiological responses to sedative music in patients awaiting cardiac catheterization examination: A randomized controlled trial. Journal of Cardiovascular Nursing, 26(5), 11-18.

Copeland, B.L., \& Franks, B.D. (1991). Effects of types and intensities of backround music on treadmil endurance. Journal of Sports Medicine and Physical Fitness, 31(1), 100-103.

Crust, L. (2008). Perceived importance of components of asynchronous music during circuit training. Journal of Sports Sciences, 26(14), 1547-1555.

Elliott, D. (1994). The effects of music and muscle relaxation on patient anxiety in a coronary care unit. Heart \& Lung, 23(1), 27-35.

Emery, C. F., Hsiao, E. T., Hill, S. M., \& Frid, D. J. (2003). Short-term effects of exercise and music on cognitive performance among participants in a cardiac rehabilitation program. Heart \& Lung, 32(6), 368-373.

Evans, D. (2002). The effectiveness of music as an intervention for hospital patients: A systematic review. Journal of Advanced Nursing, 37, 8-18.

Fletcher, G. F., Balady, G., Blair, S. N., Blumenthal, J., Caspersen, C., Chaitman, B., Pollock, M. L. (1996). Statement on exercise: Benefits and recommendations for physical activity programs for all Americans. A statement for health professionals by the Committee on Exercise and Cardiac Rehabilitation of the Council on Clinical Cardiology, American Heart Association. Circulation, 94(4), 857-862. 
Ghaderi, M., Rahimi, R., \& Ali-Azarbayjani, M. (2009). The effect of motivational and relaxation music on aerobic performance, rating perceived exertion and salivary cortisol in athlete meals. South African Journal for Research in Sport, Physical Education \& Recreation, 31(2), 29-38.

Hall, K. G., \& Erickson, B. (1995). The effects of preparatory arousal on sixty-meter dash performance. Applied Research in Coaching \& Athletics Annual, 70-79.

Hamel, W. J. (2001). The effects of music intervention on anxiety in the patient waiting for cardiac catheterization. Intensive \& Critical Care Nursing, 17(5), 279-285.

Hardy, C. J., \& Rejeski, W. J. (1989). Not what, but how one feels: The measurement of affect during exercise. Journal of Sport \& Exercise Psychology, 11(3), 304-317.

Hepler, C., \& Kapke, R. (1996). Effect of music on cardiovascular performance during treadmill walking. GAHPERD Journal, 29(2).

Hoyert, DL., \& Xu, J.Q. (2012). Deaths: Preliminary data for 2011. National Vital Statistics Reports, 61(6).

Iwanaga, M. (1995). Relationship between heart rate and preference for tempo of music. Perceptual \& Motor Skills, 81(2), 435.

Karageorghis, C., Priest, D.-L., Terry, P., Chatzisarantis, N., \& Lane, A. (2006). Redesign and initial validation of an instrument to assess the motivational qualities of music in exercise: The brunel music rating inventory-2. Journal of Sports Sciences, 24(8), 899909.

Karageorghis, C. I., Jones, L., \& Low, D. C. (2006). Relationship between exercise heart rate and music tempo preference. Research Quarterly for Exercise \& Sport, 77(2), 240-250. 
Karageorghis, C. I., Jones, L., Priest, D.-L., Akers, R. I., Clarke, A., Perry, J. M., Lim, H. B. T. (2011). Revisiting the relationship between exercise reart rate and music tempo preference. Research Quarterly for Exercise \& Sport, 82(2), 274-284.

Karageorghis, C. I., \& Priest, D.-L. (2012a). Music in the exercise domain: A review and synthesis (Part I). International Review of Sport \& Exercise Psychology, 5(1), 44-66.

Karageorghis, C. I., \& Priest, D.-L. (2012b). Music in the exercise domain: A review and synthesis (Part II). International Review of Sport \& Exercise Psychology, 5(1), 67-84.

Karageorghis, C. I., \& Terry, P. C. (1997). The psychophysical effects of music in sport and exercise: A review. Journal of Sport Behavior, 20(1), 54.

Karageorghis, C. I., Terry, P. C., \& Lane, A. M. (1999). Development and initial validation of an instrument to assess the motivational qualities of music in exercise and sport: The brunel music rating inventory. Journal of Sports Sciences, 17(9), 713-724. doi: $10.1080 / 026404199365579$

Labbé, E., Schmidt, N., Babin, J., \& Pharr, M. (2007). Coping with stress: The effectiveness of different types of music. Applied Psychophysiology \& Biofeedback, 32(3/4), 163-168.

Laukka, P. (2007). Uses of music and psychological well-being among the elderly. Journal of Happiness Studies, 8(2), 215-241.

Lee, Y. Y., Moon Fai, C., \& Mok, E. (2010). Effectiveness of music intervention on the quality of life of older people. Journal of Advanced Nursing, 66(12), 2677-2687.

Lingham, J., \& Theorell, T. (2009). Self-selected "favourite" stimulative and sedative music listening -- how does familiar and preferred music listening affect the body? Nordic Journal of Music Therapy, 18(2), 150-166. 
MacNay. (1995). The influence of preferred music on the perceived exertion, mood, and time estimation scores of patients participating in a cardiac rehabilitation program. Music Therapy Perspectives, 13, 91-96

Metzger, L. K. (2004). Assessment of use of music by patients participating in cardiac rehabilitation. Journal Of Music Therapy, 41(1), 55-69.

Mohammadzadeh, H., Tartibiyan, B., \& Ahmadi, A. (2008). The effects of music on the perceived exertion rate and performance of trained and untrained individuals during progressive exercise. Facta Universitatis: Series Physical Education \& Sport, 6(1), 6774.

Murrock, C. J. (2002). The effects of music on the rate of perceived exertion and general mood among coronary artery bypass graft patients enrolled in cardiac rehabilitation phase II. Rehabilitation Nursing, 27(6), 227-231.

Murrock, C. J., \& Higgins, P. A. (2009). The theory of music, mood and movement to improve health outcomes. Journal of Advanced Nursing, 65(10), 2249-2257.

Nakamura, P. M., Papini, C. B., Pereira, G., Nakamura, F. Y., \& Kokubun, E. (2010). Effects of preferred and nonpreferred music on continuous cycling exercise performance. Perceptual \& Motor Skills, 110(1), 257-264.

National Heart, Lung, and Blood Institute. (2012). Retrieved October 19, 2013, from http://www.nhlbi.nih.gov/health/health-topics/topics/hbp/

Nilsson, U. (2009). The effect of music intervention in stress response to cardiac surgery in a randomized clinical trial. Heart \& Lung: The Journal Of Critical Care, 38(3), 201-207.

North, A. C., \& Hargreaves, D. J. (1996). Responses to music in aerobic exercise and yogic. British Journal of Psychology, 87(4), 535. 
Özer, N., Karaman Özlü, Z., Arslan, S., \& Günes, N. (2013). Effect of music on postoperative pain and physiologic parameters of patients after open heart surgery. Pain Management Nursing, 14(1), 20-28.

Potteiger, J. A., Schroeder, J. M., \& Goff, K. L. (2000). Influence of music on ratings of perceived exertion during 20 minutes of moderate intensity exercise. Perceptual \& Motor Skills, 91(3), 848-854.

Pujol, T. J., \& Langenfeld, M. E. (1999). Influence of music on wingate anaerobic test performance. Perceptual \& Motor Skills, 88(1), 292.

Rana, S. A., Akhtar, N., \& North, A. C. (2011). Relationship between interest in music, health and happiness. Journal of Behavioural Sciences, 21(1), 48-67.

Rejeski, W. J. (1985). Perceived exertion: An active or passive process? Journal of Sport Psychology, 7(4), 371-378.

Rosch, P. J. (1994). Why zebras don't get ulcers: A guide to stress, stress-related disease and coping. Stress Medicine, 10(3), 203-203.

SAS Institute Inc. 2011. SAS/STAT® 9.3 User’s Guide. Cary, NC: SAS Institute Inc.

Schwartz, S. (1990). Effects of music on exercise performance. Journal of Cardiopulmonary Rehabilitation, 10, 312-316.

Sendelbach, S. E., Halm, M. A., Doran, K. A., Miller, E. H., \& Gaillard, P. (2006). Effects of music therapy on physiological and psychological outcomes for patients undergoing cardiac surgery. Journal of Cardiovascular Nursing, 21(3), 194-200.

Simpson, S. D., \& Karageorghis, C. I. (2006). The effects of synchronous music on 400-m sprint performance. Journal of Sports Sciences, 24(10), 1095-1102. 
Szmedra, L., \& Bacharach, D. W. (1998). Effect of music on perceived exertion, plasma lactate, norepinephrine and cardiovascular hemodynamics during treadmill running. International Journal of Sports Medicine, 19(1), 32-37.

Vaajoki, A., Kankkunen, P., Pietilä, A.-M., \& Vehviläinen-Julkunen, K. (2011). Music as a nursing intervention: Effects of music listening on blood pressure, heart rate, and respiratory rate in abdominal surgery patients. Nursing \& Health Sciences, 13(4), 412418.

Voss, J. A., Good, M., Yates, B., Baun, M. M., Thompson, A., \& Hertzog, M. (2004). Sedative music reduces anxiety and pain during chair rest after open-heart surgery. Pain (03043959), 112(1-2), 197-203.

Ware, J. E., \& Kosinski, M. (2001). Interpreting SF\&-36 summary health measures: A response. Quality of Life Research, 10(5), 405-413.

Waterhouse, J., Hudson, P., \& Edwards, B. (2010). Effects of music tempo upon submaximal cycling performance. Scandinavian Journal of Medicine \& Science in Sports, 20(4), 662669.

White, J. M. (1992). Music Therapy: An intervention to reduce anxiety in the myocardial infarction patient. Clinical Nurse Specialist, 6(2), 58-63.

White, J. M. (1999). Effects of relaxing music on cardiac autonomic balance and anxiety after acute myocardial infarction. American Journal of Critical Care, 8(4), 220-230.

Yamashita, S., Iwai, K., Akimoto, T., Sugawara, J., \& Kono, I. (2006). Effects of music during exercise on RPE, heart rate and the autonomic nervous system. Journal of Sports Medicine \& Physical Fitness, 46(3), 425-430. 
Zimmerman, L. M., Pierson, M. A., \& Marker, J. (1988). Effects of music on patient anxiety in coronary care units. Heart \& Lung, 17(5), 560-566. 


\section{CONSENT FORM - MEDICAL}

North Dakota State University - Sanford Health

Consent to Participate in a Research Study

Title: Clinical Outcomes of Different Tempos of Music During Exercise in Cardiac Rehabilitation Patients

Principal Investigator: Address:

\section{Co-Investigator(s):}

\author{
Donna J. Terbizan - NDSU .HNES Dept \\ PO Box 6050 \\ Fargo, ND 58108-6050
}

Jarad S. Miller-NDSU .HNES Dept

\section{Statement of Research}

It is a principle of medical practice that a subject who is to participate in the research investigation of a new medical treatment, device, or procedure must give his or her informed consent to such participation. This consent must be based on the understanding of the nature and risks of the treatment, device or procedure. This document provides information important for this understanding. If you have any questions, please ask. Clinical research trials include only subjects who choose to take part. Please take your time to make your decision. Discuss it with your family and friends. If at any time you have questions, please ask.

\section{Introduction}

This study is being conducted as a thesis requirement of North Dakota State University. You are being asked to participate to add knowledge to the study of music when combined with exercise. To be included into the study the criteria require that you are able to perform treadmill exercise at a walking speed of $3.0 \mathrm{mph}$, are between the ages of 55-75, and are currently enrolled in Phase 3 cardiac rehabilitation. You must meet the requirements to participate.

What is the purpose of this study?

The purpose of this study is to examine the effects of stimulating (fast tempo) and sedative (slow tempo) music when exercising.

\section{How many people will participate?}

Approximately 20 people will take part in this study at Sanford Health- Broadway Medical Center

737 N Broadway Dr, Fargo, ND 58122

(701) 234-2300

Cardiac Rehabilitation Exercise Gym

\section{How long will I be in the study?}

The study will take approximately 30 minutes each session. If you agree to take part in this study, your participation in the study will last 3 sessions covering 1-2 weeks. 


\section{What will happen during the study?}

You will be asked to participate in 10 minutes of treadmill exercise at a speed of $3.0 \mathrm{mph}$. Each participant will be asked to perform three exercise sessions each separated by $48 \mathrm{hrs}$. Recordings of blood pressure (BP), heart rate (HR), rating of perceived exertion (RPE), and feeling status will be collected throughout the exercise.

You will arrive at the hospital at your preferred workout times. After ensuring the completion of all requirements and signing an informed consent form, you will then be connected to a heart rate monitor to record heart rate and have 10 minutes of rest prior to the exercise. The investigator will then take the first recordings of all outcome measurements (BP, HR, RPE, and feeling status). Next, a music track of either stimulating music, sedative music, or a no-music control will be provided through an ipod with attaching head phones. The investigator will set the speed of the treadmill to $3.0 \mathrm{mph}$ and you will be asked perform $10 \mathrm{~min}$ of treadmill exercise under one of the music conditions.

Data collection for each exercise session will include RPE, BP, HR, and feeling status four times within the experiment. The music condition will play consistently throughout the exercise and outcome measures will be taken and recorded before, at $5 \mathrm{~min}$, at $10 \mathrm{~min}$, and after exercise. The before and after measurements will be taken 10 minutes before and 10 minutes after exercise to ensure proper resting and recovery periods have occurred. After completion of the first experimental session participants will be scheduled to return for two additional sessions.

\section{What are the risks of the study?}

There is minimal physical risk. Inclusion criteria require that you are familiar with exercise equipment, protocols, and measurements.

\section{Are there any unforeseen risks?}

In addition to the risks described, there may be unknown risks, or risks associated with being in this study, that are not anticipated.

\section{What are the benefits of this study?}

There is no direct benefit to participants in the study.

What are the alternatives to participating in this study?

Instead of being in this research study, you can choose not to participate.

\section{Will it cost me anything to be in this study?}

You will not have costs for being in this study. Your participation in this research is your choice. If you decide to participate in the study, you may change your mind and stop participating at any time without penalty or loss of benefits to which you are already entitled.

Will I be paid for participating in this study?

You will not be paid for being in this research study. 


\section{Who is funding this study?}

Sanford Health and NDSU are receiving no payments from other agencies, organizations, or companies to conduct this research.

\section{Is being in this study voluntary?}

Your participation is voluntary. You may refuse to participate or you may discontinue your participation at any time without penalty or loss of benefits to which you are otherwise entitled. Your decision whether or not to participate will not affect your current or future relations with Sanford Health or NDSU.

The investigator may also withdraw you from the study without your consent for one or more of the following reasons:

- Failure to follow the instructions of the investigator and/or study staff.

- The investigator decides that continuing your participation could be harmful to you.

- You need treatment that is not allowed in the study.

- The study is cancelled.

You will be informed by the research investigators of this study about any significant new findings that develop during the study which may influence your willingness to continue to participate in the study.

\section{Will my records be kept confidential?}

The records of this study will be kept confidential. In any report about this study that might be published, you will not be identified. Your study record may be reviewed by the Sanford Institutional Review Board (IRB) and North Dakota State Institutional Review Board. An IRB is a group of people who review the research to protect your rights. Because of the need to release information to these agencies, absolute confidentiality cannot be guaranteed, but access to records will be limited to authorized personnel as required by state or federal law.

\section{Whom may I contact if I have questions?}

You may ask any questions you have now or later.

The researchers conducting this study are:

Donna Terbizan and Jarad Miller at (701-231-7792 and 360-751-9964 respectively).

- You may call these numbers if you have questions, concerns, or complaints about the research.

- You should also call these numbers if you have a research-related injury.

If you have questions regarding your rights as a research subject, you may contact The Sanford Institutional Review Board at (605) 312-6430. An Institutional Review Board is a group of people who protect the rights and welfare of people who enroll in research.

- You may also call this number to discuss or report any problems, complaints, or concerns you have about this research study. 
- You may also call this number if you cannot reach research staff, or you wish to talk with someone who is independent of the research team.

Subject Name (Printed)

Subject's Signature

Date

\section{Statement of Person Who Obtained Consent}

Signature of Person Obtaining Consent

Date 


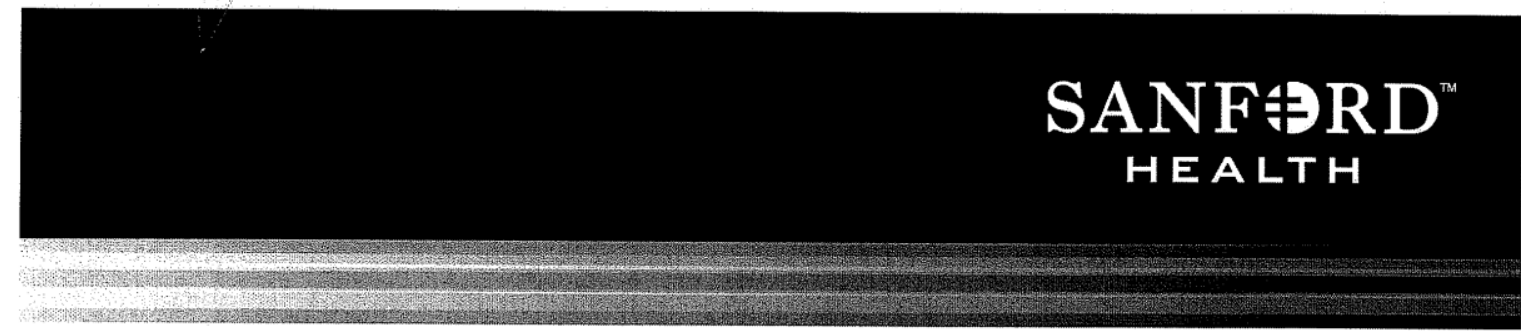

March 7, 2014

PI: Donna J. Terbizan, Ph.D., FACSM

Project: 03-14-017 Clinical Outcomes of Different Tempos of Music During Exercise in Cardiac Rehabilitation Patients

Project Review Level: Expedited 4, 7

Project Risk: No more than minimal

Amendment: Revised Consent Form dated 03/07/2014

Amendment approved through Expedited Review: 03/07/2014

Project Approval Period: 03/03/2014 - 03/02/2015

The Sanford Health Institutional Review Board (IRB) received and reviewed your amendment. The Sanford Health IRB has approved the amendment and the information has been added to the study file. Thank you for keeping the IRB informed of project changes.

Attached is your original consent document that has been stamped with the IRB approval date. You must keep this original on file. Please use this original consent document to make copies for subject enrollment/reconsent. No other consent form should be used. It must be signed by each subject prior to initiation of any protocol procedures. In addition, each subject must be given a copy of the consent form.

Prior to initiation, promptly report to the IRB, any proposed project updates / amendments (e.g., protocol amendments/revised informed consents) in previously approved human subject research activities.

The forms to assist you in filing your: project closure, continuation, adverse/unanticipated event, project updates /amendments, etc. can be accessed online at SanfordConnect.

Sincerely,

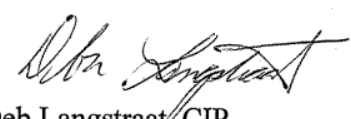

Deb Langstraat, CIP

Director-Sanford IRB 


\section{APPENDIX C. INSTITUTIONAL REVIEW BOARD LETTER}

\section{NDSU NORTHEAKROAAㄴ}

INSTITUTIONAL REVIEW BOARD

office: Research 1, 1735 NDSU Research Park Drive, Fargo, ND 58102

mail: NDSU Dept. \#4000, PO Box 6050, Fargo, ND 58108-6050

p: 701.231.8995 f: 701.231.8098 e: ndsu.irb@ndsu.edu w: www.ndsuRexw/FrWV BOARD

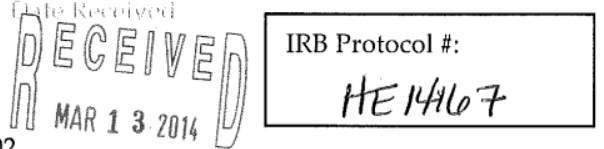

\section{Protocol Amendment Request Form}

Changes to approved research may not be initiated without prior IRB review and approval, except where necessary to eliminate apparent immediate hazards to participants. Reference: SOP 7.5 Protocol Amendments.

Examples of changes requiring IRB review include, but are not limited to changes in: investigators or research team members, purpose/scope of research, recruitment procedures, compensation strategy, participant population, research setting, interventions involving participants, data collection procedures, or surveys, measures or other data forms.

\section{Protocol Information:}

Protocol \#: HE14167 Title: Clinical Outcomes of Different Tempos of Music During Exercise in Cardiac Rehabilitation Patients

$$
\text { Review category: } \square \text { Exempt } \quad \text { Expedited } \square \text { Full board }
$$

Principal investigator: Donna J Terbizan Email address: D.Terbizan@ndsu.edu Dept: HNES

Co-investigator: Jarad S Miller Email address: Jarad.Miller@my.ndsu.edu Dept: HNES

Principal investigator signature, Date: Donna Terbitan (email) 3/13/14

- $=$ In lieu of a written signature, submission via the Principal Investigator's NDSU email constitutes an acceptable electronic signature.

Description of proposed changes:

1. Date of proposed implementation of change(s)*: $3 / 12 / 14$

* Cannot be implemented prior to IRB approval unless the IRB Chair has determined that the change is necessary to eliminate apparent immediate hazards to participants.

2. Describe proposed change(s), including justification:

We have made a change to the songs that subjects will be listening to during the exercise. The original songs that were chosen to be played for subjects are naturally fast paced songs. When the beat per minute (bpm) of the song was manipulated to meet our definition of sedative music (130 bpm) and stimulating $(90 \mathrm{bpm})$ music, we found that the music is not very motivating or likable at such altered tempos from the original version. To meet the purpose of the experiment and one of the 
main highlights in the literature, they have to like the music we're giving them. We have decided to go with naturally high or low bpm songs for the experiment instead of manipulating the bpm manually.

3. Will the change involve a change in principal or co- investigator? $\triangle$ No - skip to Question 4

Yes:

- Include an Investigator's Assurance (last page of protocol form), signed by the new PI or co-investigator

- Conflict of Interest disclosure. Does any investigator responsible for the design, conduct or reporting of the project (including their immediate family members) have a financial, personal or political interest that may conflict with their responsibility for protecting human participants in NDSU research? (SOP 6.2 Conflict of Interest in Human Research, Investigator and Research Team)

No - As PI, I attest that I have conferred with my co-investigators and key personnel and confirmed that no financial, personal or political interests currently exist related to this research.

Yes - Describe the related financial, personal or political interests, and attach documentation of COI disclosure and review (as applicable).

Financial, personal or political interests related to the research (the sponsor, product or service being tested, or a competing product or service) may include:

- compensation (e.g., salary, payment for services, consulting fees)

- intellectual property rights or equity interests

- board memberships or executive positions

- enrollment or recruitment bonus payments

(Refer to NDSU Policy 151.1, External Activities and Conflicts of Interest, and NDSU Policy 823,

Financial Disclosure - Sponsored Projects for specific disclosure requirements.)

Note: If the change is limited to addition/change in research team members, skip the rest of this form.

4. Will the change(s) increase any risks, or present new risks (physical, economic, psychological, or sociological) to participants?

$\triangle$ No

Yes: In the appropriate section of the protocol form, describe newv or altered risks and how they will be minimized.

5. Does the proposed change involve the addition of a vulnerable group of participants?

Children: $\bigotimes$ no $\square$ yes - include the Children in Research attachment form

Prisoners: $\square$ no $\square$ yes - include the Prisoners in Research attachment form

Cognitively impaired individuals: $\bigotimes$ no $\square$ yes*

Economically or educationally disadvantaged individuals: $\square$ no $\square$ yes*

*Provide additional information where applicable in the revised protocol form.

6. Does the proposed change involve a request to waive some or all the elements of informed consent or documentation of consent?

凶no 


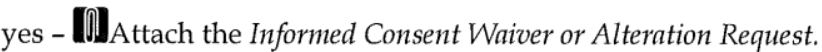

7. Does the proposed change involve a new research site?

$\bigotimes$ no

$\square$ yes

If information in your previously approved protocol has changed, or additional information is being added, incorporate the changes into relevant section(s) of the protocol. Highlight (e.g. print and highlight the hard copy, or indicate changes using all caps, asterisks, etc) the changed section(s) and attach a copy of the revised protocol to this form. (If the changes are limited to addition/change in research team members, a revised protocol form is not needed.)

\section{Impact for Participants (future, current, or prior):}

1. Will the change(s) alter information on previously approved versions of the recruitment materials, informed consent, or other documents, or require new documents?

$\bigotimes$ No

$\square$ Yes - $\square$ attach revised/new document(s)

2. Could the change(s) affect the willingness of currently enrolled participants to continue in the research? $\bigotimes$ No

$\square$ Yes - describe procedures that will be used to inform current participants, and re-consent, if necessary:

3. Will the change(s) have any impact to previously enrolled participants? $\triangle$ No

$\square$ Yes - describe impact, and any procedures that will be taken to protect the rights and welfare of participants:

\begin{tabular}{|c|c|}
\hline $\begin{array}{l}\text { Request is: } \square \longdiv { \text { Approved } \square \text { Not A } } \\
\text { Review: } \square \text { Exempt, category\#: }\end{array}$ & $\begin{array}{l}\text { pproved } \\
\square \text { Expedited method, category \# } \frac{4}{\square \text { Expedited review of minor change }} \square \text { Convened meeting, date: }\end{array}$ \\
\hline IRB Signature: Rrstyshive & Date: $3 / 13 / 4$ \\
\hline
\end{tabular}




\begin{tabular}{cc}
\hline Rating & Perceived Exertion \\
\hline 6 & No exertion \\
\hline 7 & Extremely light \\
\hline 8 & Very light \\
\hline 9 & Light \\
\hline 10 & \\
\hline 11 & Somewhat hard \\
\hline 12 & Hard \\
\hline 13 & \\
\hline 14 & Very hard \\
\hline 15 & \\
\hline 16 & Extremely hard \\
\hline 17 & Maximal exertion \\
\hline 18 & \\
\hline 19 & \\
\hline 20 & \\
\hline \hline
\end{tabular}


APPENDIX E. FEELING SCALE

$$
\begin{aligned}
+5 & \text { VERY GOOD } \\
+4 & \\
+3 & \text { GOOD } \\
+2 & \\
+1 & \text { FAIRLY GOOD } \\
0 & \text { NEUTRAL } \\
-1 & \text { FAIRLY BAD } \\
-2 & \\
-3 & \text { BAD } \\
-4 & \\
-5 & \text { VERY BAD }
\end{aligned}
$$

